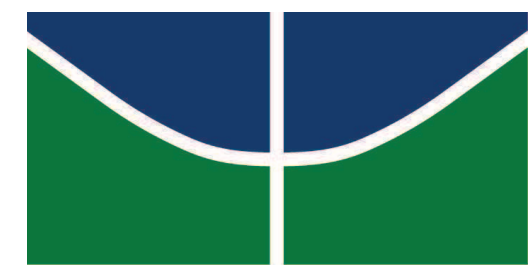

Universidade de Brasília

Instituto de Ciências Exatas

Departamento de Matemática

\title{
Uma propriedade das álgebras de Grassmann não-unitárias sobre um corpo de característica prima e suas aplicações
}

por

Bruno Trindade Reis 
Uma propriedade das álgebras de Grassmann nãounitárias sobre um corpo de característica prima e suas aplicações.

por

\section{Bruno Trindade Reis}

Tese apresentada ao Corpo Docente do Programa de Pós-Graduação em Matemática-UnB, como requisito parcial para obtenção do grau de

\section{DOUTOR EM MATEMÁTICA}

Brasília, 30 de junho de 2016.

Comissão Examinadora:

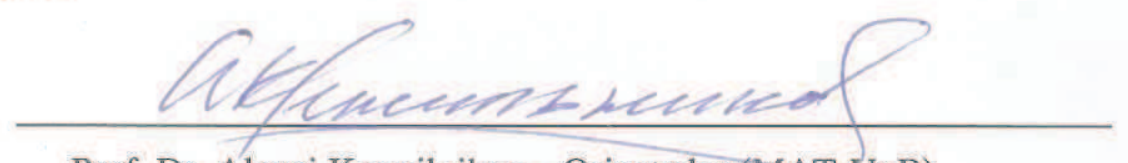

Prof. Dr. Alexei Krassilnikov - Orientador (MAT-UnB)

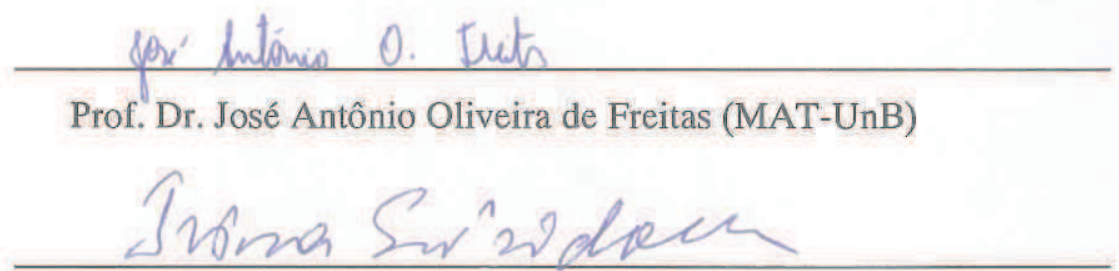

Profa. Dra. Irina Sviridova (MAT-UnB)

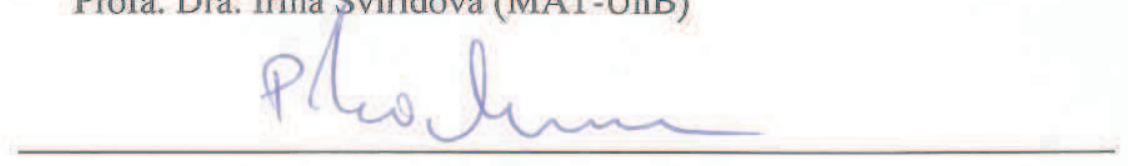

Prof. Dr. Plamen Emilov Kochloukov (UNICAMP)

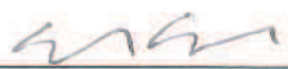

Profa. Dra. Shirlei Serconek (UFG)

\footnotetext{
* O autor foi bolsista CNPq durante a elaboração desta tese.
} 
Ficha catalográfica elaborada automaticamente, com os dados fornecidos pelo(a) autor(a)

Reis, Bruno Trindade

Uma propriedade das álgebras de Grassmann não unitárias sobre um corpo de característica prima e suas aplicações / Bruno Trindade Reis; orientador Alexei Krassilnikov. -- Brasília, 2016. $66 \mathrm{p}$.

Tese (Doutorado - Doutorado em Matemática) - Universidade de Brasília, 2016.

1. Álgebra de Grassmann. 2. Identidades polinomiais. 3. Álgebras livres. 4. Álgebras graduadas. I. Krassilnikov, Alexei, orient. II. Título. 
À minha família. 
"Se as portas da percepção estivessem limpas, tudo se mostraria ao homem tal como é: infinito". (William Blake) 


\section{Agradecimentos}

Agradeço à minha família, pelo apoio. À minha esposa Renata, que esteve sempre ao meu lado. Agradeço ao meu orientador, Professor Alexei Krassilnikov, por ter me orientado e por ter confiado no meu trabalho.

Agradeço a todos os meus amigos, pelo companheirismo: Alex Teló, Benedito, Agenor, Sílvio, Adriano, Claud, Eudes, Raimundo, Marcos Duarte, Aramis, Daiane, Ludimila, Hiuri, Laís, Gustavo Tiveron e todos que contribuíram para essa conquista. 


\section{Resumo}

Seja $K$ um corpo de característica $p>2$. Sejam $H$ a álgebra de Grassmann nãounitária de dimensão infinita e $H_{n}$ a álgebra de Grassmann não unitária de um espaço vetorial de dimensão finita $n$, ambas sobre $K$. Seja $\mathcal{A}=K\langle Y, Z\rangle / T_{2}(H)$ a álgebra relativamente livre $\mathbb{Z}_{2}$-graduada da variedade de $K$-álgebras associativas $\mathbb{Z}_{2}$-graduadas nãounitárias determinada por $H$. Seja $\mathcal{D}=K\langle X\rangle / T(H)$ a álgebra relativamente livre da variedade de álgebras associativas não-unitárias (sem graduação) determinada por $H$. Nesse trabalho construímos um mergulho de $\mathcal{A}$ em $H$, que determina um mergulho de $\mathcal{D}$ em $H$. Isso nos permite dar demonstrações simples e unificadas de resultados sobre identidades polinomiais e polinômios centrais de $H$ e $H_{n}$ obtidos anteriormente por vários autores. Os resultados obtidos também são válidos se $K$ é um domínio de integridade de característica $p>2$.

Estudamos também a álgebra de Grassmann unitária $E$ de dimensão infinita sobre um corpo finito. Seja $K$ um corpo finito e $K_{1}\langle X\rangle$ a álgebra associativa livre unitária, livremente gerada por $X$. Damos uma representação de $K_{1}\langle X\rangle / T(E)$ como produto tensorial da álgebra comutativa $A=K\left[t_{i} \mid i \in \Lambda\right] / I$, onde $I$ é o ideal de $K\left[t_{i} \mid i \in \Lambda\right]$ gerado por $t_{i}^{q}-t_{i}, i \in \Lambda$, e a álgebra $B=K_{1}\langle Y\rangle / V$, onde $V$ é o $T$-ideal de $K\langle Y\rangle$ (ou seja, da álgebra associativa livre não-unitária) gerado por $y_{1}^{p}$ e pelo comutador triplo $\left[y_{1}, y_{2}, y_{3}\right]$. Essa representação nos permite dar uma demonstração mais simples do resultado de Bekh-Ochir e Rankin sobre uma base de identidades polinomiais de $E$ sobre um corpo finito.

Palavras-chave: Álgebra de Grassmann; Identidades polinomiais; Álgebras livres; Álgebras $\mathbb{Z}_{2}$-graduadas. 


\section{Abstract}

Let $K$ be a field of characteristic $p>2$. Let $H$ be the infinite dimensional nonunitary Grassmann algebra and $H_{n}$ the non-unitary Grassmann algebra of a vector space of dimension $n$, both over $K$. Let $\mathcal{A}=K\langle Y, Z\rangle / T_{2}(H)$ be the $\mathbb{Z}_{2}$-graded relatively free algebra of the variety of $\mathbb{Z}_{2}$-graded non-unitary associative algebras determined by $H$. Let $\mathcal{D}=K\langle X\rangle / T(H)$ be the relatively free algebra of the variety of non-unitary associative algebras (without grading) determined by $H$. In this work we construct an embedding of $\mathcal{A}$ in $H$, determining an embedding of $\mathcal{D}$ in $H$. This allows us to give simple and unified proofs of results about polynomial identities and central polynomials of $H$ e $H_{n}$ obtained previously by several authors. The results obtained are also valid if $K$ is an integral domain of characteristic $p>2$.

We study also the infinite dimensional unitary Grassmann algebra $E$ over a finite field. Let $K$ be a finite field and $K_{1}\langle X\rangle$ the unitary associative free algebra, freely generated by $X$. We give a representation of $K_{1}\langle X\rangle / T(E)$ as a tensor product of the commutative algebra $A=K\left[t_{i} \mid i \in \Lambda\right] / I$, where $I$ is the ideal of $K\left[t_{i} \mid i \in \Lambda\right]$ generated by $t_{i}^{q}-t_{i}$, $i \in \Lambda$, and the algebra $B=K_{1}\langle Y\rangle / V$, where $V$ is the $T$-ideal of $K\langle Y\rangle$ (that is, of the free associative non-unitary algebra) generated by $y_{1}^{p}$ and $\left[y_{1}, y_{2}, y_{3}\right]$. This representation allows us to give a simple proof of the result of Bekh-Ochir and Rankin on a basis of the polynomial identities of $E$ over a finite field.

Keywords: Grassmann algebras; Polynomial identities; Free algebras; $\mathbb{Z}_{2}$-graded algebras. 


\section{Sumário}

1 Introdução $\quad 9$

2 Preliminares $\quad 15$

2.1 PI-álgebras . . . . . . . . . . . . . . . . . . . . . . . . . . . 15

2.2 Álgebras $\mathbb{Z}_{2}$-Graduadas e álgebras de Grassmann . . . . . . . . . . . . . . 22

3 Álgebra de Grassmann não-unitária de dimensão infinita 28

3.1 Identidades $\mathbb{Z}_{2}$-graduadas de $H \quad \ldots \ldots \ldots \ldots \ldots$

3.2 Identidades de $H \ldots \ldots \ldots$. . . . . . . . . . . . . . . . . . . . . . 31

3.3 Polinômios Centrais de $H \ldots \ldots \ldots$. . . . . . . . . . . . . 35

4 Álgebra de Grassmann não-unitária de dimensão finita 45

4.1 Identidades $\mathbb{Z}_{2}$-graduadas de $H_{n} \ldots \ldots \ldots \ldots \ldots$. . . . . . . . 45

4.2 Identidades de $H_{2 n} \quad \ldots \ldots \ldots \ldots \ldots$

4.3 Identidades de $H_{2 n-1} \ldots \ldots \ldots \ldots$. . . . . . . . . . . . . . . . . . . 53

5 Álgebra de Grassmann unitária de dimensão infinita $\quad 58$

$\begin{array}{ll}\text { Referências Bibliográficas } & 64\end{array}$ 


\section{Capítulo 1}

\section{Introdução}

A álgebra de Grassmann aparece naturalmente em muitos campos da matemática e da física. Ela é muito importante na teoria de álgebras com identidades polinomiais (PIálgebras). Mais precisamente, sejam $X$ um conjunto infinito enumerável, $K$ um corpo e $K\langle X\rangle$ a álgebra livre não unitária sobre $K$, livremente gerada por $X$. Dada uma $K$ álgebra $R$, dizemos que um polinômio $f\left(x_{1}, \ldots, x_{n}\right)$ de $K\langle X\rangle$ é uma identidade polinomial para $R$ se $f\left(a_{1}, \ldots, a_{n}\right)=0$ para todos $a_{1}, \ldots, a_{n} \in R$. Se a álgebra $R$ satisfaz uma identidade polinomial não-trivial, dizemos que $R$ é uma PI-álgebra. Para uma introdução à PI-teoria, ver, por exemplo, [10, 11, 13, 20, 22, 29].

Dada uma $K$-álgebra $R$, uma das importantes questões da PI-teoria é descrever as identidades de $R$. Outra questão é saber se $R$ possui uma base finita para suas identidades polinomiais. Para álgebras sobre um corpo $K$ de característica zero, esse problema foi proposto por Specht [33]. Um famoso resultado de Kemer [21], [23] (ver também [22]) deu uma solução afirmativa para o problema de Specht (para álgebras sobre um corpo de característica zero). Por outro lado, para álgebras associativas sobre um corpo $K$ de característica $p>0$, o análogo do problema de Specht tem solução negativa, ou seja, existem álgebras sobre $K$ cujas identidades polinomiais não possuem nenhuma base finita. Isso foi demonstrado em 1999, independentemente por Belov [5], Grishin [16] e Shchigolev [31] (ver também [4], [18], [32]). No caso em que $K$ tem característica $p>2$, as demonstrações foram baseadas no resultado de Shchigolev [30] sobre T-subespaços não finitamente gerados em álgebras que satisfazem as identidades da álgebra de Grassmann unitária $E$ de dimensão infinita. Mais ainda, como foi descoberto depois em [1], [6] e [17], o resultado de Shchigolev é equivalente ao fato de que o $T$-subespaço de polinômios centrais da álgebra $E$ não é finitamente gerado. 
Latyshev [26] provou que sobre um corpo de característica zero, o T-ideal da álgebra associativa livre unitária $K_{1}\langle X\rangle$ gerado por $\left[x_{1}, x_{2}, x_{3}\right]$ tem uma base finita. Em [25], Krakowski e Regev encontraram uma base para as identidades da álgebra de Grassmann unitária $E$ de dimensão infinita sobre um corpo de característica zero. Eles mostraram que as identidades da álgebra de Grassamnn $E$ sobre tal corpo seguem do comutador $\left[x_{1}, x_{2}, x_{3}\right]$. Observamos que as identidades da álgebra de Grassmann não-unitária de dimensão infinita sobre um corpo de característica zero também seguem do comutador $\left[x_{1}, x_{2}, x_{3}\right]$. Para a álgebra de Grassmann unitária de dimensão infinita sobre um corpo infinito de característica $p>2$, vários autores (ver, por exemplo, Giambruno e Koshlukov [12]) mostraram que toda identidade segue do comutador $\left[x_{1}, x_{2}, x_{3}\right]$. Bekh-Ochir e Rankin, em [3], estudaram as identidades da álgebra $E$ de dimensão infinita sobre um corpo finito. Nesse caso, as identidades da álgebra de Grassmann segue do comutador $\left[x_{1}, x_{2}, x_{3}\right]$ e do polinômio $x_{1}^{p q}-x_{1}^{p}$, onde $p$ e $q$ são a característica e o número de elementos do corpo, respectivamente. Em [34] Stojanova-Venkova exibiu uma base para as identidades da álgebra de Grassmann não-unitária de um espaço vetorial de dimensão finita sobre um corpo de característica prima. Chiripov e Siderov em [8] encontraram uma base para as identidades da álgebra de Grassmann não unitária de dimensão infinita $H$ sobre um corpo de característica $p>2$. Essa base é formanda pelos polinômios $\left[x_{1}, x_{2}, x_{3}\right]$ e $x_{1}^{p}$. Em [6], [2] e [17], independentemente, foram descritos os polinômios centrais para a álgebra de Grassmann unitária e não-unitária de dimensão infinita sobre um corpo de característica diferente de 2 .

Observamos que foram publicados vários trabalhos sobre as identidades e os polinômios centrais da álgebra de Grassmann unitária e assuntos relacionados, ver, por exemplo, $[1,2,3,6,7,12,14,15,17,19,25,28,36]$. Por outro lado, as identidades polinomiais e os polinômios centrais da álgebra de Grassmann não-unitária foram estudados com menos frequência, ver, por exemplo, [2, 6, 8, 24, 28, 34]. Observamos também que as demonstrações dos resultados no caso da álgebra de Grassmann não-unitária são em geral mais complicadas que no caso da álgebra de Grassmann unitária. No nosso trabalho, vamos apresentar versões mais simples dessas demonstrações.

Começamos com o seguinte: Sejam $K$ um domínio de integridade de característica $p>2, K_{1}\langle X\rangle$ a álgebra associativa livre unitária e $K\langle X\rangle$ a álgebra associativa livre nãounitária. Apesar do título desse trabalho, vamos considerar o caso mais geral quando $K$ é domínio de integridade. No segundo capítulo, definiremos os conceitos básicos para o entendimento do restante desse trabalho. Enunciaremos e demonstraremos alguns resultados sobre álgebras livres e PI-álgebras sobre um domínio de integridade $K$. A seguir, 
apresentaremos vários resultados sobre álgebras $\mathbb{Z}_{2}$-graduadas e identidades $\mathbb{Z}_{2}$-graduadas. Definiremos álgebra de Grassmann e veremos como ela possui uma $\mathbb{Z}_{2}$-graduação natural. Veremos também como as identidades $\mathbb{Z}_{2}$-graduadas podem ser utilizadas para encontrar as identidades ordinárias de uma álgebra.

No terceiro capítulo, demonstraremos nosso principal resultado. Sejam $K$ um domínio de integridade de característica $p>2$ e $H$ a álgebra de Grassmann não-unitária de dimensão infinita sobre $K$. Seja $\mathcal{D}=K\langle X\rangle / T(H)$ a álgebra relativamente livre da variedade de álgebras associativas determinada por $H$.

Teorema 1.1. A álgebra $\mathcal{D}$ pode ser mergulhada em $H$, isto é, $\mathcal{D} \subset H$.

Esse mergulho permite dar uma demonstração simples do seguinte resultado já obtido por um método mais complicado por Chiripov e Siderov [8] quando $K$ é um corpo.

Corolário 1.2. Seja $H$ a álgebra de Grassmann não-unitária de dimensão infinita sobre um dominio de integridade de característica $p>2$. O conjunto das identidades de $H$ é $o$ $T$-ideal de $K\langle X\rangle$ gerado por $\left[x_{1}, x_{2}, x_{3}\right]$ e $x^{p}$.

Obtemos também de forma simples uma $K$-base para o quociente $K\langle X\rangle / T(H)$, a mesma base que foi descrita por Kireeva e Krasilnikov em [24] com uma demonstração mais complicada, quando $K$ é um corpo.

Corolário 1.3. Seja $K$ um domínio de integridade de característica $p>2$. O quociente $K\langle X\rangle / T(H)$ é um $K$-módulo livre com uma $K$-base formada pelos polinômios

$$
x_{i_{1}}^{m_{1}} \cdots x_{i_{k}}^{m_{k}}\left[x_{j_{1}}, x_{j_{2}}\right] \cdots\left[x_{j_{2 l-1}}, x_{j_{2 l}}\right]+T(H)
$$

onde $k \geq 0, l \geq 0, k+l>0, i_{1}<\ldots<i_{k}, j_{1}<\ldots<j_{2 l}, 0<m_{i}<p$.

Para mostrar isso, trabalharemos com a álgebra de Grassmann $H=H_{0} \oplus H_{1}$ vista como álgebra $\mathbb{Z}_{2}$-graduada. Sejam $K\langle Y, Z\rangle$ a álgebra livre não-unitária $\mathbb{Z}_{2}$-graduada e $T_{2}$ o $T_{2}$-ideal de $K\langle Y, Z\rangle$ gerado pelos seguintes polinômios

$$
\left[y_{1}, y_{2}\right], \quad\left[y_{1}, z_{1}\right], \quad z_{1} z_{2}+z_{2} z_{1}, \quad y_{1}^{p}
$$

Começaremos demonstrando o seguinte: 
Teorema 1.4. Seja K um dominio de integridade de caracteristica $p>2$. A álgebra relativamente livre $\mathbb{Z}_{2}$-graduada da variedade determinada por $T_{2}$ pode ser mergulhada em $H$, isto é, $K\langle Y, Z\rangle / T_{2} \subset H$.

Mais precisamente, dividimos os geradores de $H$ em duas famílias

$$
e_{1}, e_{2}, \ldots ; f_{1}, f_{2}, \ldots
$$

A seguir mostramos que o homomorfismo

$$
\begin{aligned}
& \phi: K\langle Y, Z\rangle / T_{2} \longrightarrow H \\
& z_{i}+T_{2} \mapsto f_{i} \\
& y_{i}+T_{2} \mapsto e_{\lambda_{i}+1} e_{\lambda_{i}+2}+e_{\lambda_{i}+3} e_{\lambda_{i}+4}+\ldots+e_{\lambda_{i}+(2 p-3)} e_{\lambda_{i}+(2 p-2)},
\end{aligned}
$$

onde $\lambda_{i}=(i-1)(2 p-2)$, é um homomorfismo injetor.

Esse teorema implica dois resultados que provavelmente são bem conhecidos quando $K$ é um corpo, embora não tenhamos encontrado as referências.

Corolário 1.5. O conjunto das identidades $\mathbb{Z}_{2}$-graduadas de $H$ é o $T_{2}$-ideal gerado pelos seguintes polinômios de $K\langle Y, Z\rangle$ :

$$
\left[y_{1}, y_{2}\right], \quad\left[y_{1}, z_{1}\right], \quad z_{1} z_{2}+z_{2} z_{1}, \quad y_{1}^{p}
$$

Corolário 1.6. Seja $K$ um dominio de integridade de característica $p>2$. O quociente $K\langle Y, Z\rangle / T_{2}(H)$ é um $K$-módulo livre com uma $K$-base formada pelos monômios

$$
y_{i_{1}}^{m_{1}} \cdots y_{i_{k}}^{m_{k}} z_{j_{1}} \cdots z_{j_{l}}+T_{2}(H)
$$

onde $i_{1}<\ldots<i_{k}, j_{1}<\ldots<j_{l} ; 0<m_{1}, \ldots, m_{k}<p ; k, l \geq 0$ e $k+l>0$.

Usaremos esses dois últimos corolários para demonstrar os corolários 1.2 e 1.3. Como $\mathcal{D}$ é isomorfo à subálgebra de $K\langle Y, Z\rangle / T_{2}(H)$ gerada por $x_{i}+T_{2}(H)$, onde $x_{i}=y_{i}+z_{i}$, trabalharemos com $\mathcal{D}$ como subálgebra do quociente $K\langle Y, Z\rangle / T_{2}(H)$. Mostraremos que se $V$ é o $T$-ideal de $K\langle X\rangle$ gerado pelos polinômios $\left[x_{1}, x_{2}, x_{3}\right]$ e $x_{1}^{p}$, então $\mathcal{D} \simeq K\langle X\rangle / V$.

A seguir, usando os resultados de [6] encontraremos uma base para o $T$-subespaço de polinômios centrais de $H$. Obtemos também uma nova demonstração simples do fato 
de que o $T$-subespaço de polinômios centrais de $H$ não é finitamente gerado como um T-espaço. Esse resultado foi demonstrado de modo mais complicado em [1] e [6] usando o resultado sofisticado de Shchigolev [30]. A importância desse resultado se deve ao fato que a partir de $C(H)$ foram construídos exemplos de $T$-ideais não finitamente gerados obtidos por Belov [20], Grishin [17] e Shchigolev [30]. Esses exemplos resolveram o análogo do problema de Specht para álgebras sobre corpos de característica $p>2$ que ficou em aberto por um longo período.

No capítulo 4, estudaremos as álgebras de Grassmann de um $K$-módulo livre de dimensão $n, H_{n}$, sobre um domínio de integridade de característica $p>2$. Seguiremos o mesmo caminho do capítulo 3. Primeiro, encontraremos uma base para as identidades $\mathbb{Z}_{2}$-graduadas de $H_{n}$ e uma $K$-base para o quociente $K\langle Y, Z\rangle / T_{2}\left(H_{n}\right)$. Como $H_{n} \subset H$, temos que $H_{n}$ satisfaz as identidades (1.2). Como o grau dos elementos da $K$-base de $H_{n}$ é limitado, é de se esperar que monômios de $K\langle Y, Z\rangle$ com grau suficientemente grande sejam identidades de $H_{n}$. Para isso, definiremos peso de um monômio de $K\langle Y, Z\rangle$ como sendo a soma dos pesos das variáveis que compôem o monômio, onde as variáveis $Y=\left\{y_{1}, y_{2}, \ldots\right\}$ tem peso igual a 2 e as variáveis $Z=\left\{z_{1}, z_{2}, \ldots\right\}$ tem peso igual a 1 . Assim temos o seguinte teorema, que provavelmente é bem conhecido quando $K$ é um corpo.

Teorema 1.7. Seja K um dominio de integridade de caracteristica $p>2$. O quociente $K\langle Y, Z\rangle / T_{2}\left(H_{n}\right)$ é um $K$-módulo livre com uma $K$-base formada pelos monômios, módulo $T_{2}\left(H_{n}\right)$,

$$
y_{i_{1}}^{m_{1}} \ldots y_{i_{k}}^{m_{k}} z_{j_{1}} \ldots z_{j_{l}}
$$

onde $2\left(m_{1}+\cdots+m_{k}\right)+l \leq n, k+l>0, k, l \geq 0 i_{1}<\cdots<i_{k}, j_{1}<\cdots<j_{l}$, $0<m_{1}, \ldots, m_{k}<p$.

Assim todo monômio de $K\langle Y, Z\rangle$ com peso maior do que $n$ é uma identidade $\mathbb{Z}_{2^{-}}$ graduada para $H_{n}$. Para encontrar uma $K$-base para $K\langle X\rangle / T\left(H_{n}\right)$ e o $T$-ideal $T\left(H_{n}\right)$, teremos que considerar dois casos separadamente: $n$ é par e $n$ ímpar. Isso foi feito em [34] por Stojanova-Venkova quando $K$ é um corpo de característica $p>2$. Seja $x_{1} \circ x_{2}=x_{1} x_{2}+x_{2} x_{1} \in K\langle X\rangle$. Definimos o polinômios $v_{n+1}$ do seguinte modo:

$$
v_{n+1}=\left(\cdots\left(\left(x_{1} \circ x_{2}\right) \circ x_{3}\right) \circ \ldots \circ x_{n+1}\right) \circ x_{n+1} .
$$

Então temos 
Teorema 1.8. Seja $H_{2 n}$ a álgebra de Grassmann de um $K$-módulo livre de dimensão $2 n$, não-unitária, sobre um dominio de integridade de característica $p>2$. O T-ideal das identidades de $\mathrm{H}_{2 n}$ é o T-ideal gerado pelos polinômios

$$
\left[x_{1}, x_{2}, x_{3}\right], x_{1}^{p} \text { e } v_{n+1}
$$

Teorema 1.9. Seja $H_{2 n-1}$ a álgebra de Grassmann de um K-módulo livre de dimensão $2 n-1$, não-unitária, sobre um dominio de integridade de caracteristica $p>2$. O T-ideal das identidades de $H_{2 n-1}$ é o T-ideal gerado pelo polinômios

$$
\left[x_{1}, x_{2}, x_{3}\right], x_{1}^{p}, v_{n} x_{n+1}, x_{n+1} v_{n}
$$

Se $s=n /(2 p-1)$ é um número inteiro, incluímos o polinômio

$$
\left[x_{1}, x_{2}\right] \cdots\left[x_{2 s-1}, x_{2 s}\right] x_{1}^{p-1} \cdots x_{2 s}^{p-1} .
$$

No capítulo 5, consideraremos a álgebra de Grassmann unitária $E$, de um espaço de dimensão infinita, sobre um corpo finito $K$. Esse caso foi estudado por Bekh-Ochir e Rankin em [3]. Nesse artigo eles mostraram que o $T$-ideal das identidades de $E$ é o $T$-ideal gerado pelos polinômios $\left[x_{1}, x_{2}, x_{3}\right]$ e $x_{1}^{q p}-x_{1}^{p}$, onde $p$ é a característica do corpo $K$ e $q$ é a quantidade de elementos de $K$. O principal teorema desse capítulo consiste no seguinte. Seja $U$ o $T$-ideal de $K_{1}\langle X\rangle$ gerado por $\left[x_{1}, x_{2}, x_{3}\right]$ e $x_{1}^{q p}-x_{1}^{p}$, então

Teorema 1.10. $K_{1}\langle X\rangle / U \simeq A \bigotimes_{K} B$, onde $A=K_{1}\left[t_{i} \mid i \in \Lambda\right] / I$ e $B=K_{1}\langle Y\rangle / V$. Aqui, I é o ideal de $K_{1}\left[t_{i} \mid i \in \Lambda\right]$ gerado por $t_{i}^{q p}-t_{i}^{p}, i \in \Lambda$, e $V$ é o $T$-ideal de $K\langle Y\rangle$ gerado por $\left[y_{1}, y_{2}, y_{3}\right]$ e $y_{1}^{p}$.

Por fim, concluiremos pelo teorema acima que o $T$-ideal das identidades de $E$ é $U$, isto é, $U=T(E)$, dando assim uma demonstração mais simples do resultado de Bekh-Ochir e Rankin [3]. 


\section{Capítulo 2}

\section{Preliminares}

Neste capítulo, recordaremos alguns resultados de álgebras associativas livres, álgebras com identidades polinomiais e álgebras $\mathbb{Z}_{2}$-graduadas que serão importantes para nosso trabalho. Neste capítulo, a menos que dito o contrário, $K$ será um domínio de integridade.

\section{$2.1 \quad$ PI-álgebras}

Começamos definindo álgebras associativas livres e álgebras relativamente livres.

Definição 2.1. Sejam $\mathfrak{D}$ uma classe de álgebras associativas sobre um dominio de integridade $K$ e $F \in \mathfrak{D}$ uma álgebra gerada por um conjunto $X$. A álgebra $F$ é chamada livre na classe $\mathfrak{D}$, livremente gerada pelo conjunto $X$, se para qualquer álgebra $R \in \mathfrak{D}$, toda aplicação $\phi: X \rightarrow R$ pode ser estendida a um homomorfismo $\bar{\phi}: F \rightarrow R$. A cardinalidade $|X|$ do conjunto $X$ é chamada posto de $F$.

Se $\mathfrak{D}$ é a classe de todas as álgebras associativas, então $F$ é simplesmente chamada de álgebra associativa livre. Caso contrário, $F$ é chamada de álgebra associativa relativamente livre na classe $\mathfrak{D}$.

Sejam $K$ um corpo e $X$ um conjunto infinito enumerável. A álgebra $K_{1}\langle X\rangle$ tendo como base o conjunto

$$
x_{i_{1}} x_{i_{2}} \cdots x_{i_{n}}, \quad x_{i_{l}} \in X, n=0,1,2,3 \ldots
$$


e multiplicação definida por

$$
\left(x_{i_{1}} x_{i_{2}} \cdots x_{i_{n}}\right)\left(x_{j_{1}} x_{j_{2}} \cdots x_{j_{m}}\right)=x_{i_{1}} x_{i_{2}} \cdots x_{i_{n}} x_{j_{1}} x_{j_{2}} \cdots x_{j_{m}}
$$

é livre na classe de todas as álgebras associativas unitárias.

Seja $K\langle X\rangle$ o $K$-submódulo de $K_{1}\langle X\rangle$ gerado por todas as palavras $x_{i_{1}} x_{i_{2}} \cdots x_{i_{n}}$, $x_{i_{l}} \in X, n=1,2,3 \ldots$, isto é, todas as palavras com comprimento maior ou igual a 1 . Então $K\langle X\rangle$ é livre na classe de todas as álgebras associativas não-unitárias.

Agora podemos definir identidades polinomiais de uma álgebra associativa.

Definição 2.2. Seja $f\left(x_{1}, \ldots, x_{n}\right) \in K\langle X\rangle$ e $R$ uma álgebra associativa sobre $K$. Dizemos que $f=0$ é uma identidade polinomial para $R$ se $f\left(r_{1}, \ldots, r_{n}\right)=0$ para todos $r_{1}, \ldots, r_{n} \in R$.

Diremos que $f=0$ é uma identidade para $R$ (ou simplesmente $f$ é identidade para $R$ ), ou $R$ satisfaz $f$.

Definição 2.3. Se $R$ satisfaz uma identidade polinomial não trivial, dizemos que $R$ é uma PI-álgebra.

Exemplo 2.4. Se $R$ é uma K-álgebra associativa de dimensão $n$, então $R$ satisfaz a identidade standard de grau $n+1$,

$$
s_{n+1}=\sum_{\sigma \in S_{n+1}} \operatorname{sign}(\sigma) x_{\sigma(1)} \cdots x_{\sigma(n+1)} \text {. }
$$

Exemplo 2.5 (Amitsur-Levitzki). A álgebra de matrizes de ordem $n, M_{n}(K)$, satisfaz o polinômio standard de grau $2 n, s_{2 n}=\sum_{\sigma \in S_{2 n}} \operatorname{sign}(\sigma) x_{\sigma(1)} \cdots x_{\sigma(2 n)}$.

Seja $\Phi$ o conjunto de todos os homomorfismos $\phi: K\langle X\rangle \longrightarrow R$. Então é claro que $f=0$ é uma identidade polinomial para $R$ se, e somente se, $f \in \bigcap_{\phi \in \Phi} \operatorname{Ker} \phi$.

Definição 2.6. Sejam $R$ uma $K$-álgebra com centro $Z(R)$ e seja $f\left(x_{1}, \ldots, x_{n}\right) \in K\langle X\rangle$. $O$ polinômio $f$ é central em $R$ se $f\left(r_{1}, \ldots, r_{n}\right) \in Z(R)$ para todos $r_{1}, \ldots, r_{n} \in R$.

Dada uma álgebra $R$, denotaremos por $C(R)$ o conjunto de polinômios centrais de $R$. 
Dada uma álgebra $R$, seja

$$
T(R)=\{f \in K\langle X\rangle \mid f \quad \text { é identidade para } R\}
$$

o conjunto de todas as identidades polinomiais de $R$. Claramente, $T(R)$ é um ideal bilateral de $K\langle X\rangle$. Além disso, se $f\left(x_{1}, \ldots, x_{n}\right) \in T(R)$ e $g_{1}, \ldots, g_{n}$ são polinômios arbitrários de $K\langle X\rangle$, então $f\left(g_{1}, \ldots, g_{n}\right) \in T(R)$. Como todo endomorfismo de $K\langle X\rangle$ é determinado por aplicações $x \mapsto g, x \in X, g \in K\langle X\rangle$, segue que $T(R)$ é fechado por todos os endomorfismos de $K\langle X\rangle$. Ideais com essa propriedade são chamados de $T$-ideais.

Definição 2.7. Um ideal $I$ de $K\langle X\rangle$ é um $T$-ideal se $\phi(I) \subseteq I$ para todos os endomorfismos $\phi$ de $K\langle X\rangle$.

Portanto, para toda álgebra $R, T(R)$ é um $T$-ideal de $K\langle X\rangle$. Por outro lado, todo $T$-ideal $I$ é conjunto de identidades polinomiais de alguma álgebra, pois $T(K\langle X\rangle / I)=I$.

Analogamente define-se um $T$-subespaço como sendo um $K$-submódulo de $K\langle X\rangle$ fechado por todos os endomorfismos de $K\langle X\rangle$. Embora a palavra $T$-subespaços seja usada quando $K$ é um corpo, isto é, para $K$-subespaços fechados por todos os endomorfismos de $K\langle X\rangle$, aqui ela será usada no mesmo sentido.

Definição 2.8. Um $K$-submódulo $J$ de $K\langle X\rangle$ é um $T$-subespaço se $\phi(J) \subseteq J$ para todos os endomorfismos $\phi$ de $K\langle X\rangle$.

Temos também que para toda álgebra $R$, o conjunto de polinômios centrais $C(R)$ é um $T$-subespaço de $K\langle X\rangle$. Mas a recíproca não vale.

Seja $S=\left\{f_{i} \in K\langle X\rangle \mid i \in I\right\}$ um conjunto de polinômios. O $T$-ideal de $K\langle X\rangle$ gerado por $S$ será denotado por $\langle S\rangle^{T}$. É fácil ver que $\langle S\rangle^{T}$ é o ideal gerado por

$$
f_{i}\left(g_{1}, \ldots, g_{n_{i}}\right)
$$

onde $f_{i}\left(x_{1}, \ldots, x_{n_{i}}\right) \in S$ e $g_{1}, \ldots, g_{n_{i}}$ são polinômios arbitrários de $K\langle X\rangle$.

Vimos que toda álgebra $R$ determina um $T$-ideal de $K\langle X\rangle$. Introduziremos agora o conceito de variedade de álgebras.

Definição 2.9. Seja $\left\{f_{i} \in K\langle X\rangle \mid i \in \Lambda\right\}$ um conjunto de polinômios de $K\langle X\rangle$. A classe de todas as álgebras $R$ tais que $f_{i}=0, i \in \Lambda$, são identidades polinomiais para $R$ é chamada de variedade determinada por $\left\{f_{i} \in K\langle X\rangle \mid i \in \Lambda\right\}$. 
Note que se $S=\left\{f_{i} \in K\langle X\rangle \mid i \in \Lambda\right\}$, a variedade determinada por $S$ é igual a variedade determinada por $\langle S\rangle^{T}$.

O próximo teorema fornece um critério para saber se uma dada classe de álgebras é uma variedade.

Teorema 2.10 (Birkhoff). Uma classe de álgebras $\mathfrak{D}$ é uma variedade se, e somente se, $\mathfrak{D}$ é fechada para produtos diretos, subálgebras e álgebras quocientes.

Seja $\mathcal{V}$ a variedade determinada por um $T$-ideal $I$. A próxima proposição, que pode ser encontrada em [10], nos mostra a existência de uma álgebra relativamente livre na classe $\mathcal{V}$.

Proposição 2.11. Seja $K\langle X\rangle$ a álgebra associativa livre, livremente gerada pelo conjunto $X$. Seja $\mathcal{V}$ a variedade determinada por um $T$-ideal $I \in K\langle X\rangle$. Então $K\langle X\rangle / I$ é uma álgebra relativamente livre, livremente gerada por $\bar{X}=\{x+I \mid x \in X\}$, na classe $\mathcal{V}$. Além disso, duas álgebras relativamente livres na classe $\mathcal{V}$, de mesmo posto, são isomorfas.

Demonstração. Sejam $R$ uma álgebra que pertence à variedade $\mathcal{V}$ e uma aplicação $\phi: \bar{X} \rightarrow R$ onde $\phi(x+I)=r, r \in R$. Defina uma aplicação $\psi: X \rightarrow R$ colocando $\psi(x)=\phi(x+I)$. Como $K\langle X\rangle$ é álgebra livre sobre $X, \psi$ pode ser estendida a um homomorfismo $\bar{\psi}: K\langle X\rangle \rightarrow R$, onde $\bar{\psi}\left(f\left(x_{1}, \ldots, x_{n}\right)\right)=f\left(r_{1}, \ldots, r_{n}\right)$. Mas $I \subseteq T(R) \subseteq \operatorname{ker}(\bar{\psi})$. Assim o homomorfismo

$$
\begin{aligned}
& K\langle X\rangle / I \rightarrow R \\
& \bar{\phi}\left(f\left(x_{1}, \ldots, x_{n}\right)+I\right)=f\left(r_{1}, \ldots, r_{n}\right)
\end{aligned}
$$

está bem definido e estende $\phi$.

Sejam $R_{1}, R_{2} \in \mathcal{V}$ álgebras relativamente livres de mesmo posto sobre $X=\left\{x_{1}, x_{2}, \ldots\right\}$ e $X^{\prime}=\left\{x_{1}^{\prime}, x_{2}^{\prime}, \ldots\right\}$, respectivamente. Como $R_{1}$ e $R_{2}$ são álgebras relativamente livres na variedade $\mathcal{V}$, existem homomorfismos $\phi_{1}: R_{1} \rightarrow R_{2}$ e $\phi_{2}: R_{2} \rightarrow R_{1}$ tais que $\phi_{1}\left(x_{i}\right)=x_{i}^{\prime}$ e $\phi_{2}\left(x_{i}^{\prime}\right)=x_{i}$ para $i=1,2, \ldots$ É fácil ver que $\phi_{1} \phi_{2}$ é a aplicação identidade de $X^{\prime}$ e $\phi_{2} \phi_{1}$ é a aplicação identidade de $X$. Portanto, $R_{1}$ e $R_{2}$ são isomorfas.

A proposicão acima diz que se $R$ pertence a variedade $\mathcal{V}$, então toda aplicação

$$
\begin{aligned}
\bar{X} & \rightarrow R \\
x_{i}+I & \mapsto g_{i},
\end{aligned}
$$


pode ser estendida a um homomorfismo

$$
K\langle X\rangle / I \longrightarrow R
$$

Vamos agora introduzir os conceitos de identidades multi-homogêneas, identidades multilineares.

Definição 2.12. Dizemos que um polinômio $f\left(x_{1}, \ldots, x_{n}\right) \in K\langle X\rangle$ é homogêneo com respeito à variável $x_{1}$, se ele pode ser representado como combinação linear de monômios de mesmo grau, relativamente a $x_{1}$.

Definição 2.13. Um polinômio $f\left(x_{1}, \ldots, x_{n}\right) \in K\langle X\rangle$ é multi-homogêneo se for homogêneo com respeito a todas as variáveis.

Definição 2.14. Um polinômio multi-homogêneo $f\left(x_{1}, \ldots, x_{n}\right) \in K\langle X\rangle$ tem multi-grau $\left(d_{1}, \ldots, d_{n}\right)$ se o grau de $x_{i}$ em $f$ é $d_{i}, i=1, \ldots, n$.

Assim cada $f\left(x_{1}, \ldots, x_{n}\right) \in K\langle X\rangle$ pode ser escrito de maneira única como combinação linear de suas componentes multi-homogêneas,

$$
f=\sum_{d_{1} \geq 0, \ldots, d_{n} \geq 0} f^{\left(d_{1}, \ldots, d_{n}\right)}
$$

onde $f^{\left(d_{1}, \ldots, d_{n}\right)}$ é a componente multi-homogênea de $f$ com multi-grau $\left(d_{1}, \ldots, d_{n}\right)$. Portanto $K\langle X\rangle$ é graduada pelos seus multi-graus, isto é,

$$
K\langle X\rangle=\bigoplus_{d=\left(d_{1}, \ldots, d_{n}\right)} K\langle X\rangle^{\left(d_{1}, \ldots, d_{n}\right)}
$$

onde $K\langle X\rangle^{\left(d_{1}, \ldots, d_{n}\right)}$ é o $K$-submódulo de $K\langle X\rangle$ gerado por todos os polinômios com multi$\operatorname{grau}\left(d_{1}, \ldots, d_{n}\right)$.

Definição 2.15. Um polinômio $f\left(x_{1}, \ldots, x_{n}\right) \in K\langle X\rangle$ é multilinear se o grau com respeito a cada variável $x_{1}, \ldots, x_{n}$ é um.

Definição 2.16. Um polinômio $g \in K\langle X\rangle$ é consequência dos polinômios $f_{i} \in K\langle X\rangle$, $i \in \Lambda$, se $g \in\left\langle f_{i} \mid i \in \Lambda\right\rangle^{T}$.

Definição 2.17. Dois sistemas de identidades, $S_{1}, S_{2}$, são equivalentes se eles geram o mesmo T-ideal, isto é, $\left\langle S_{1}\right\rangle^{T}=\left\langle S_{2}\right\rangle^{T}$. 
Um importante teorema relacionado com identidades multi-homogêneas é o seguinte. Ele pode ser encontrado por exemplo em [10] e [13].

Proposição 2.18. Seja

$$
f\left(x_{1}, \ldots, x_{n}\right)=\sum_{i=0}^{m} f_{i} \in K\langle X\rangle
$$

onde $f_{i}$ é a componente homogênea de $f$ de grau $i$ em $x_{1}$. Se existe um corpo contido em $K$ com mais que $m$ elementos, então os polinômios $f_{i}, i=0,1, \ldots, m$, são consequências de $f$.

Demonstração. Seja $I=\langle f\rangle^{T}$ o $T$-ideal de $K\langle X\rangle$ gerado por $f$. Como $K$ possui um corpo com mais de $m$ elementos, escolhemos $m+1$ elementos distintos desse corpo, $\alpha_{0}, \ldots, \alpha_{m}$. Como $I$ é um $T$-ideal,

$$
f\left(\alpha_{j} x_{1}, \ldots, x_{n}\right)=\sum_{i=1}^{m} \alpha_{j}^{i} f_{i}\left(x_{1}, \ldots, x_{n}\right) \in I, \quad j=0,1, \ldots, m
$$

Escrevendo essas $m$ equações na forma matricial, temos

$$
\left(\begin{array}{c}
f\left(\alpha_{0} x_{1}, \ldots, x_{n}\right) \\
f\left(\alpha_{1} x_{1}, \ldots, x_{n}\right) \\
f\left(\alpha_{2} x_{1}, \ldots, x_{n}\right) \\
\vdots \\
f\left(\alpha_{m} x_{1}, \ldots, x_{n}\right)
\end{array}\right)=\left(\begin{array}{ccccc}
1 & \alpha_{0} & \alpha_{0}^{2} & \cdots & \alpha_{0}^{m} \\
1 & \alpha_{1} & \alpha_{1}^{2} & \cdots & \alpha_{1}^{m} \\
1 & \alpha_{2} & \alpha_{2}^{2} & \cdots & \alpha_{2}^{m} \\
\vdots & \vdots & \vdots & \ddots & \vdots \\
1 & \alpha_{m} & \alpha_{m}^{2} & \cdots & \alpha_{m}^{m}
\end{array}\right)\left(\begin{array}{c}
f_{0}\left(x_{1}, \ldots, x_{n}\right) \\
f_{1}\left(x_{1}, \ldots, x_{n}\right) \\
f_{2}\left(x_{1}, \ldots, x_{n}\right) \\
\vdots \\
f_{m}\left(x_{1}, \ldots, x_{n}\right)
\end{array}\right)
$$

O determinante

$$
\left|\begin{array}{ccccc}
1 & \alpha_{0} & \alpha_{0}^{2} & \cdots & \alpha_{0}^{m} \\
1 & \alpha_{1} & \alpha_{1}^{2} & \cdots & \alpha_{1}^{m} \\
1 & \alpha_{2} & \alpha_{2}^{2} & \cdots & \alpha_{2}^{m} \\
\vdots & \vdots & \vdots & \ddots & \vdots \\
1 & \alpha_{m} & \alpha_{m}^{2} & \cdots & \alpha_{m}^{m}
\end{array}\right|=\prod_{i<j}\left(\alpha_{j}-\alpha_{i}\right)
$$

é o determinante de Vandermonde e é diferente de zero. Assim, pela equação (2.1), podemos escrever cada $f_{i}\left(x_{1}, \ldots, x_{n}\right)$ como uma combinação linear dos polinômios $f\left(\alpha_{j} x_{1}, \ldots, x_{n}\right)$, $i=0, \ldots, m$. Logo, cada $f_{i}\left(x_{1}, \ldots, x_{n}\right)$ pertence a $I$, isto é, as identidades $f_{i}$ são consequências de $f$. 
Uma das consequências mais importantes desse teorema é que sobre um corpo infinito todo $T$-ideal é gerado pelos seus polinômios multi-homogêneos. Assim temos a seguinte definição.

Definição 2.19. Um ideal $I$ de $K\langle X\rangle$ é multi-homogêneo se para todo $f \in I$, todas as componentes multi-homogêneas de $f$ também pertencem a $I$.

Analogamente define-se um $K$-submódulo multi-homogêneo de $K\langle X\rangle$.

Vimos que $K\langle X\rangle$ pode ser escrito como soma direta de $K$-submódulos

$$
K\langle X\rangle=\bigoplus_{d=\left(d_{1}, \ldots, d_{n}\right)} K\langle X\rangle^{\left(d_{1}, \ldots, d_{n}\right)}
$$

onde $K\langle X\rangle^{\left(d_{1}, \ldots, d_{n}\right)}$ é o $K$-submódulo de $K\langle X\rangle$ gerado por todos os polinômios com multigrau $\left(d_{1}, \ldots, d_{n}\right)$. Para uma álgebra quociente $K\langle X\rangle / I$, onde $I$ é um $T$-ideal de $K\langle X\rangle$, definimos o seguinte.

Definição 2.20. Uma álgebra quociente $K\langle X\rangle / I$, onde $I$ é um $T$-ideal de $K\langle X\rangle$, é graduada pelos seus multi-graus se

$$
K\langle X\rangle / I=\bigoplus_{d=\left(d_{1}, \ldots, d_{n}\right)} K\langle X\rangle^{\left(d_{1}, \ldots, d_{n}\right)}+I
$$

onde $K\langle X\rangle^{\left(d_{1}, \ldots, d_{n}\right)}+I$ é o $K$-submódulo de $K\langle X\rangle / I$ gerado por todos $f+I \in K\langle X\rangle / I$ onde $f$ tem multi-grau $\left(d_{1}, \ldots, d_{n}\right)$.

Com isso, temos a seguinte proposição.

Proposição 2.21. Um ideal $I$ de $K\langle X\rangle$ é multi-homogêneo se, e somente se, $K\langle X\rangle / I$ é graduado pelos seus multi-graus.

Demonstração. Suponha que $I$ não é multi-homogêneo. Então existe $f \in I$ tal que pelo menos duas componentes multi-homogêneas de $f$ não pertencem a $I$. Suponha, por exemplo, que $f^{\left(d_{1}, \ldots, d_{k}\right)}$ e $f^{\left(d_{1}^{\prime}, \ldots, d_{k^{\prime}}^{\prime}\right)}$ não pertencem a $I$ e que todas as outras componentes multi-homogêneas de $f$ pertencem a $I$. Assim,

$$
\begin{aligned}
f+I=I & \Rightarrow f^{\left(d_{1}, \ldots, d_{k}\right)}+f^{\left(d_{1}^{\prime}, \ldots, d_{k^{\prime}}^{\prime}\right)}+I=I \\
& \Rightarrow\left(f^{\left(d_{1}, \ldots, d_{k}\right)}+I\right)+\left(f^{\left(d_{1}^{\prime}, \ldots, d_{k^{\prime}}^{\prime}\right)}+I\right)=I
\end{aligned}
$$


Logo, a soma de $K\langle X\rangle^{\left(d_{1}, \ldots, d_{n}\right)}+I$ e $K\langle X\rangle^{\left(d_{1}^{\prime}, \ldots, d_{n^{\prime}}^{\prime}\right)}+I$ não é direta. Assim, $K\langle X\rangle / I$ não é graduada pelos seus multi-graus. O mesmo raciocínio é válido supondo que mais de duas componentes multi-homogêneas de $f$ não pertencem a $I$.

Agora suponha que $K\langle X\rangle / I$ não é graduada pelos seus multi-graus. Assim, existem polinômios multi-homogêneos, não nulos em $K\langle X\rangle / I, f^{\left(d_{1}, \ldots, d_{k}\right)}+I$ e $f^{\left(d_{1}^{\prime}, \ldots, d_{k^{\prime}}^{\prime}\right)}+I$, tais que

$$
f^{\left(d_{1}, \ldots, d_{k}\right)}+I=f^{\left(d_{1}^{\prime}, \ldots, d_{k^{\prime}}^{\prime}\right)}+I .
$$

Logo, $f^{\left(d_{1}, \ldots, d_{k}\right)}-f^{\left(d_{1}^{\prime}, \ldots, d_{k^{\prime}}^{\prime}\right)} \in I$. Como $f^{\left(d_{1}, \ldots, d_{k}\right)} \notin I$ e $f^{\left(d_{1}^{\prime}, \ldots, d_{k^{\prime}}^{\prime}\right)} \notin I$, temos que $I$ não é multi-homogêneo.

\section{2 Álgebras $\mathbb{Z}_{2}$-Graduadas e álgebras de Grassmann}

Introduziremos nessa seção os conceitos de álgebras associativas $\mathbb{Z}_{2}$-graduadas e álgebras de Grassmann. Começaremos definindo álgebras de Grassmann.

Definição 2.22. Sejam $X$ um conjunto infinito enumerável, $K$ um dominio de integridade de característica $p>2$ e $K\langle X\rangle$ a álgebra associativa livre não-unitária, livremente gerada por $X$. Seja I o ideal bilateral de $K\langle X\rangle$ gerado pelos polinômios $\left\{x_{i} x_{j}+x_{j} x_{i} \mid i, j \geq 1\right\}$. A álgebra $H=K\langle X\rangle / I$ é chamada álgebra de Grassmann, não-unitária, de um K-módulo livre de dimensão infinita. Analogamente define-se a álgebra de Grassmann unitária como $E=K_{1}\langle X\rangle / I$, onde $I$ é o ideal bilateral de $K_{1}\langle X\rangle$ gerado por $\left\{x_{i} x_{j}+x_{j} x_{i} \mid i, j \geq 1\right\}$.

Se escrevermos $e_{i}=x_{i}+I, i=1,2, \ldots$, então $H$ tem a seguinte apresentação:

$$
H=\left\langle e_{1}, e_{2}, \ldots \mid e_{i} e_{j}=-e_{j} e_{i}, i, j \geq 1\right\rangle \text {. }
$$

Uma base de $H$ como $K$-espaço vetorial é o conjunto

$$
\left\{e_{i_{1}} e_{i_{2}} \cdots e_{i_{k}} \mid i_{1}<i_{2}<\cdots<i_{k}, k>0\right\} .
$$

Definição 2.23. A subálgebra $H_{n}$ da álgebra $H$, gerada por $e_{1}, e_{2}, \ldots, e_{n}$, é chamada de álgebra de Grassmann, não-unitária, de um K-módulo livre de dimensão $n$. 
Logo, $H_{n}$ tem uma apresentação

$$
H_{n}=\left\langle e_{1}, e_{2}, \ldots, e_{n} \mid e_{i} e_{j}=-e_{j} e_{i}, 1 \leq i, j \leq n\right\rangle
$$

e tem como uma $K$-base o conjunto

$$
\left\{e_{i_{1}} e_{i_{2}} \cdots e_{i_{k}} \mid 1 \leq i_{1}<i_{2}<\cdots<i_{k} \leq n\right\}
$$

A seguir, definiremos álgebras $G$-graduadas, onde $G$ é um grupo, e veremos que $H$ tem uma $\mathbb{Z}_{2}$-graduação natural.

Definição 2.24. Seja $G$ um grupo. Uma $K$-álgebra $R$ é G-graduada se admite uma decomposição como uma soma direta $R=\bigoplus_{g \in G} R_{g}$, onde cada $R_{g}$ é um K-submódulo de $R$ e $R_{g_{1}} R_{g_{2}} \subseteq R_{g_{1} g_{2}}$, para todos $g_{1}, g_{2} \in G$. Os submódulos $R_{g}$ são chamados submódulos homogêneos de $R$.

Definição 2.25. Um ideal I de uma álgebra G-graduada $R$ é um ideal G-graduado se

$$
I=\bigoplus_{g \in G} I_{g}, \text { onde } I_{g}=I \cap R_{g}
$$

Seja $I$ um ideal $G$-graduado de uma álgebra $G$-graduada $R$. A álgebra $R / I$ também é uma álgebra $G$-graduada, com $(R / I)_{g}=\left\{r+I \mid r \in R_{g}\right\}$ sendo seus submódulos homogêneos.

Definição 2.26. Um homomorfismo $\phi: R \rightarrow R^{\prime}$ entre álgebras G-graduadas é $G$ graduado se $\phi\left(R_{g}\right) \subseteq R_{g}^{\prime}$ para todo $g \in G$.

A álgebra de Grassmann $H$ é uma álgebra $\mathbb{Z}_{2}$-graduada. De fato, seja

$$
H_{0}=\operatorname{span}\left\{e_{i_{1}} e_{i_{2}} \cdots e_{i_{2 k}} \mid i_{1}<i_{2}<\cdots<i_{k}, k>0\right\}
$$

e

$$
H_{1}=\operatorname{span}\left\{e_{i_{1}} e_{i_{2}} \cdots e_{i_{2 k+1}} \mid i_{1}<i_{2}<\cdots<i_{k}, k \geq 0\right\}
$$

Assim, $H=H_{0} \oplus H_{1}$. O submódulo $H_{0}$ é chamado de parte par de $H$ e $H_{1}$ de parte ímpar. 
Sejam $Y$ e $Z$ conjuntos infinitos enumeráveis disjuntos e seja $K$ um domínio de integridade de característica $p>2$. A álgebra livre $K\langle Y, Z\rangle$, livremente gerada por $Y \cup Z$, é chamada de álgebra livre $\mathbb{Z}_{2}$-graduada. De fato, $K\langle Y, Z\rangle$ tem uma $\mathbb{Z}_{2}$-graduação natural

$$
K\langle Y, Z\rangle=K\langle Y, Z\rangle_{0} \oplus K\langle Y, Z\rangle_{1}
$$

onde $K\langle Y, Z\rangle_{0}$ (respectivamente $K\langle Y, Z\rangle_{1}$ ) é o subespaço gerado por todos os monômios que contem um número par (respectivamente ímpar) de variáveis de $Z$.

Agora podemos definir identidades polinomiais $\mathbb{Z}_{2}$-graduadas.

Definição 2.27. Seja $f\left(y_{1}, \ldots, y_{n}, z_{1}, \ldots, z_{m}\right) \in K\langle Y, Z\rangle$ e seja $R=R_{0} \bigoplus R_{1}$ uma álgebra $\mathbb{Z}_{2}$-graduada. O polinômio $f$ é uma identidade polinomial $\mathbb{Z}_{2}$-graduada da álgebra $R$ se $f\left(r_{1}, \ldots, r_{n}, r_{1}^{\prime}, \ldots, r_{m}^{\prime}\right)=0$ para todos $r_{1}, \ldots, r_{n} \in R_{0}$ e $r_{1}^{\prime}, \ldots, r_{m}^{\prime} \in R_{1}$.

Exemplo 2.28. A álgebra de Grassmann $H$ satisfaz as identidades $\mathbb{Z}_{2}$-graduadas $\left[y_{1}, y_{2}\right]$ $e\left[y_{1}, z_{1}\right]$, pois os elementos de $H_{0}$ são centrais em $H$.

As seguintes proposições mostram que $H$ satisfaz outras identidades $\mathbb{Z}_{2}$-graduadas.

Proposição 2.29. A álgebra de Grassmann $H$ satisfaz a identidade $\mathbb{Z}_{2}$-graduada $z_{1} z_{2}+z_{2} z_{1}$.

Demonstração. O resultado segue da igualdade

$$
e_{i_{1}} e_{i_{2}} \cdots e_{i_{k}} e_{j_{1}} e_{j_{2}} \cdots e_{j_{l}}=(-1)^{k l} e_{j_{1}} e_{j_{2}} \cdots e_{j_{l}} e_{i_{1}} e_{i_{2}} \cdots e_{i_{k}}
$$

De fato, todo elemento de $H_{1}$ é combinação linear de monômios de comprimento ímpar e se $k$ e $l$ são ímpares, então $l k$ é ímpar.

Proposição 2.30. A álgebra de Grassmann $H$ satisfaz a identidade $y_{1}^{p}$.

Demonstração. Seja $u=m_{1}+m_{2}$, onde $m_{1}$ e $m_{2}$ são monômios de comprimento par em H. Como $m_{1}$ e $m_{2}$ comutam temos

$$
\begin{aligned}
u^{p} & =\left(m_{1}+m_{2}\right)^{p} \\
& =m_{1}^{p}+\alpha_{1} m_{1}^{p-1} m_{2}+\ldots+\alpha_{p-1} m_{1} m_{2}^{p-1}+m_{2}^{p},
\end{aligned}
$$

onde $\alpha_{i}=\left(\begin{array}{c}p \\ i\end{array}\right), i=1, \ldots, p-1$. Como a característica de $K$ é $p$ e os $\alpha_{i}$ são múltiplos de $p$, temos que $u^{p}=m_{1}^{p}+m_{2}^{p}=0$. Procedendo por indução, seja $u=m_{1}+\ldots+m_{k} \in H$, 
$k>2$ onde $m_{1}, \ldots, m_{k}$ são monômios de comprimento par em $H$. Suponha que $\left(u^{\prime}\right)^{p}=0$, onde $u^{\prime}=m_{1}+\ldots+m_{k-1}$. Assim

$$
\begin{aligned}
u^{p} & =\left(u^{\prime}+m_{k}\right)^{p} \\
& =\left(u^{\prime}\right)^{p}+m_{k}^{p} \\
& =0 .
\end{aligned}
$$

Como todo elemento de $H_{0}$ é combinação linear de monômios de comprimento par, o resultado segue.

Agora, como $K\langle Y, Z\rangle$ é a álgebra associativa livre $\mathbb{Z}_{2}$-graduada, dada uma álgebra $\mathbb{Z}_{2}$-graduada $R=R_{0} \bigoplus R_{1}$, toda aplicação

$$
\begin{aligned}
Y \cup Z & \rightarrow R \\
y_{i} & \mapsto g_{i}^{(0)} \\
z_{i} & \mapsto g_{i}^{(1)}
\end{aligned}
$$

onde $g_{i}^{(0)} \in R_{0}$ e $g_{i}^{(1)} \in R_{1}$, pode ser estendida de maneira única a um homomorfismo

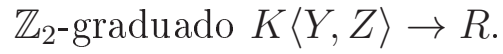

Definição 2.31. Seja I um ideal bilateral $\mathbb{Z}_{2}$-graduado de $K\langle Y, Z\rangle$. O ideal I é um $T_{2}$-ideal se $\phi(I) \subseteq I$ para todo endomorfismo $\mathbb{Z}_{2}$-graduado de $K\langle Y, Z\rangle$.

Dada uma álgebra $\mathbb{Z}_{2}$-graduada $R$, seja

$$
T_{2}(R)=\left\{f \in K\langle Y, Z\rangle \mid f \text { é identidade } \mathbb{Z}_{2} \text {-graduada de } R\right\} .
$$

O ideal $T_{2}(R)$ é um $T_{2}$-ideal. Também $T_{2}(R)=\bigcap_{\phi \in \Omega} \operatorname{ker}(\phi)$, onde $\Omega$ é o conjunto de todos os homomorfismos $\mathbb{Z}_{2}$-graduados de $K\langle Y, Z\rangle$ em $R$.

Definição 2.32. Seja I um $T_{2}$-ideal de $K\langle Y, Z\rangle$. A variedade de álgebras $\mathbb{Z}_{2}$-graduadas determinada por $I$ é a classe de todas as álgebras $\mathbb{Z}_{2}$-graduadas $R$ tais que $I \subseteq T_{2}(R)$.

Em álgebras $\mathbb{Z}_{2}$-graduadas, também temos a existência de álgebra relativamente livre $\mathbb{Z}_{2 \text {-graduada. }}$ 
Proposição 2.33. Seja $K\langle Y, Z\rangle$ a álgebra associativa livre $\mathbb{Z}_{2}$-graduada. Seja $\mathcal{V}_{I}$ a variedade determinada por um $T_{2}$-ideal $I \in K\langle Y, Z\rangle$. Então $K\langle Y, Z\rangle / I$ é uma álgebra relativamente livre $\mathbb{Z}_{2}$-graduada, livremente gerada por $\bar{Y} \cup \bar{Z}=\{y+I, z+I \mid y \in Y, z \in Z\}$, na classe $\mathcal{V}_{I}$.

Demonstração. A demonstração é análoga ao caso de álgebras não-graduadas.

Logo, se $R$ é uma álgebra $\mathbb{Z}_{2}$-graduada que pertence à variedade $\mathcal{V}_{I}$, então toda aplicação

$$
\begin{gathered}
\bar{Y} \cup \bar{Z} \rightarrow R \\
y_{i}+I \mapsto g_{i}^{(0)} \\
z_{i}+I \mapsto g_{i}^{(1)}
\end{gathered}
$$

onde $g_{i}^{(0)} \in R_{0}, g_{i}^{(1)} \in R_{1}$, pode ser estendida a um homomorfismo de álgebras $\mathbb{Z}_{2}$-graduadas $K\langle Y, Z\rangle / I \rightarrow R$.

O próximo teorema mostra como o conhecimento das identidades $\mathbb{Z}_{2}$-graduadas pode ajudar a encontrar as identidade ordinárias de uma dada álgebra $R$.

Teorema 2.34. Sejam $R=R_{0} \oplus R_{1}$ uma álgebra $\mathbb{Z}_{2}$-graduada e $T_{2}(R)$ o $T_{2}$-ideal das identidades de $R$. Seja $\mathcal{D}$ a subálgebra de $K\langle Y, Z\rangle / T_{2}(R)$ gerada por $x_{i}+T_{2}(R)$ onde $x_{i}=y_{i}+z_{i}$. Então $\mathcal{D}$ é a álgebra relativamente livre da variedade determinada por $R$, isto é, $\mathcal{D} \simeq K\langle X\rangle / T(R)$.

Demonstração. Sejam $T_{2}=T_{2}(R)$ e $X+T_{2}=\left\{x_{i}+T_{2}\right\}$. Primeiro vamos mostrar que toda aplicação $X+T_{2} \rightarrow R$ pode ser estendida a um homomorfismo $\mathcal{D} \rightarrow R$. Suponha então que temos uma aplicação

$$
\begin{aligned}
\theta: X+T_{2} & \rightarrow R \\
x_{i}+T_{2} & \mapsto g_{i}
\end{aligned}
$$

Como $g_{i} \in R, g_{i}=g_{i}^{(0)}+g_{i}^{(1)}$, onde $g_{i}^{(0)}$ e $g_{i}^{(1)}$ pertencem a parte par e ímpar de $R$, respectivamente. Defina o homomorfismo $\mathbb{Z}_{2}$-graduado

$$
\begin{gathered}
\phi: K\langle Y, Z\rangle / T_{2} \rightarrow R \\
y_{i}+T_{2} \mapsto g_{i}^{(0)} \\
z_{i}+T_{2} \mapsto g_{i}^{(1)}
\end{gathered}
$$


A restrição de $\phi$ a $\mathcal{D},\left.\phi\right|_{\mathcal{D}}$, é um homomorfismo. Além disso, $\phi\left(x_{i}+T_{2}\right)=g_{i}$, isto é, $\left.\phi\right|_{\mathcal{D}}$ estende $\theta$.

Vemos que todo homomorfismo de $\mathcal{D} \rightarrow R$ é restrição de um homomorfismo $\mathbb{Z}_{2}$ graduado $K\langle Y, Z\rangle / T_{2} \rightarrow R$. Também, todo homomorfismo $K\langle Y, Z\rangle / T_{2} \rightarrow R$ define um homomorfismo $\mathcal{D} \rightarrow R$. Logo,

$$
\bigcap_{\theta: \mathcal{D} \rightarrow R} \operatorname{Ker}(\phi) \subseteq \bigcap_{\phi: K\langle Y, Z\rangle / T_{2} \rightarrow R} \operatorname{Ker}(\phi)=0
$$

onde $\{\theta: \mathcal{D} \rightarrow R\}$ são todos os homomorfismos de $\mathcal{D}$ em $R$ e $\left\{\phi: K\langle Y, Z\rangle / T_{2} \rightarrow R\right\}$ são todos homomorfismos $\mathbb{Z}_{2}$-graduados de $K\langle Y, Z\rangle / T_{2}$ em $R$. Note que essa última igualdade é zero porque $K\langle Y, Z\rangle / T_{2}$ é a álgebra relativamente livre $\mathbb{Z}_{2}$-graduada da variedade determinada por $R$. Mas

$$
\bigcap_{\theta: \mathcal{D} \rightarrow R} \operatorname{Ker}(\phi)=0
$$

implica que $\mathcal{D} \simeq K\langle X\rangle / T(R)$. 


\section{Capítulo 3}

\section{Álgebra de Grassmann não-unitária de dimensão infinita}

Neste capítulo, estudaremos a álgebra de Grassmann não-unitária de dimensão infinita sobre um domínio de integridade $K$ de característica prima $p>2$. Demonstraremos nosso resultado principal, que diz que a álgebra relativamente livre da variedade de álgebras associativas determinada por $T(H)$ pode ser mergulhada em $H$. Faremos isso exibindo um homomorfismo $\mathbb{Z}_{2}$-graduado injetor de $K\langle Y, Z\rangle / T_{2}(H)$ em $H$. A seguir, se $\mathcal{D}$ é a subálgebra de $K\langle Y, Z\rangle / T_{2}(H)$ gerada por $x_{i}+T_{2}(H)$, onde $x_{i}=y_{i}+z_{i}$, então $\mathcal{D} \simeq$ $K\langle X\rangle / T(H)$, isto é, $\mathcal{D}$ é a álgebra relativamente livre da variedade determinada por $T(H)$. Mostraremos que a subálgebra $\mathcal{D}$ pode ser mergulhada em $H$. Usando esse mergulho, encontraremos de forma simples um conjunto gerador para o $T$-ideal das identidades ordinárias de $H$ e uma $K$-base para o $K$-módulo livre $K\langle X\rangle / T(H)$, resultados já obtidos em [8] e [24], respectivamente, quando $K$ é um corpo.

\section{$3.1 \quad$ Identidades $\mathbb{Z}_{2}$-graduadas de $H$}

Seja $K$ um domínio de integridade de característica prima $p>2$. Sejam $Y$ e $Z$ conjuntos infinitos enumeráveis disjuntos e $K\langle Y, Z\rangle$ a $K$-álgebra associativa livre $\mathbb{Z}_{2}$-graduada. Definimos $\mathcal{A}=K\langle Y, Z\rangle / T_{2}$, onde $T_{2}$ é o $T_{2}$-ideal de $K\langle Y, Z\rangle$ gerado pelos seguintes polinômios:

$$
\left[y_{1}, y_{2}\right], \quad\left[y_{1}, z_{1}\right], \quad z_{1} z_{2}+z_{2} z_{1}, \quad y_{1}^{p}
$$


É fácil verificar que um conjunto gerador de $\mathcal{A}$ visto como $K$-módulo é formado pelos monômios

$$
y_{i_{1}}^{m_{1}} \cdots y_{i_{k}}^{m_{k}} z_{j_{1}} \cdots z_{j_{l}}+T_{2}
$$

onde $i_{1}<\ldots<i_{k}, j_{1}<\ldots<j_{l} ; 0<m_{1}, \ldots, m_{k}<p ; k, l \geq 0$ e $k+l>0$. De fato, uma $K$-base do $K$-módulo livre $K\langle Y, Z\rangle$ é formada por todos os monômios em $Y$ e $Z$. As relações $\left[y_{1}, y_{2}\right]$ e $\left[y_{1}, z_{1}\right]$ significam que os elementos $y_{i}+T_{2}$ são centrais em $\mathcal{A}$ e a relação $z_{1} \circ z_{2}$ implica que os elementos $z_{i}+T_{2}$ anti-comutam e que $z_{i}^{2}+T_{2}=0$. Portanto se $u=u\left(y_{i_{1}}, \ldots, y_{i_{k}}, z_{j_{1}}, \ldots, z_{j_{l}}\right)$ é um monômio de $K\langle Y, Z\rangle$, então podemos reordenar as variáveis de $u+T_{2}$ de modo que

$$
u+T_{2}=y_{i_{1}}^{m_{1}} \cdots y_{i_{k}}^{m_{k}} z_{j_{1}} \cdots z_{j_{l}}+T_{2},
$$

onde $i_{1}<\ldots<i_{k}, j_{1}<\ldots<j_{l} ; 0<m_{1}, \ldots, m_{k}<p ; k, l \geq 0$ e $k+l>0$.

Agora escreveremos uma base para $H$ visto como $K$-módulo livre da seguinte forma. Dividimos os geradores de $H$ em dois subconjuntos

$$
e_{1}, e_{2}, \ldots ; f_{1}, f_{2}, \ldots
$$

Definimos a seguinte ordem nos geradores:

$$
e_{1}<e_{2}<e_{3}<\ldots ; f_{1}<f_{2}<f_{3}<\ldots ; e_{i}<f_{j} \text {, para todos } i, j \text {. }
$$

Logo, o conjunto

$$
\left\{e_{i_{1}} e_{i_{2}} \cdots e_{i_{k}} f_{j_{1}} f_{j_{2}} \cdots f_{j_{l}} \mid i_{1}<\ldots<i_{k}, j_{1}<\ldots<j_{l}, k+l>0\right\}
$$

forma uma base para $H$ visto como $K$-módulo livre.

No capítulo 2 vimos que $H$, como álgebra $\mathbb{Z}_{2}$-graduada, satisfaz as identidades (3.1). Assim, $H$ pertence à variedade determinada por $T_{2}$. Vamos demonstrar o seguinte teorema (ver introdução).

Teorema 1.4 Seja K um dominio de integridade de caracteristica $p>2$. A álgebra relativamente livre $\mathbb{Z}_{2}$-graduada $\mathcal{A}$, da variedade determinada por $T_{2}$, pode ser imersa em $H$, isto é, $\mathcal{A} \subset H$. 
Demonstração. Demonstraremos esse teorema exibindo um homomorfismo $\mathbb{Z}_{2}$-graduado injetor de $\mathcal{A}$ em $H$. Seja

$$
\begin{aligned}
& \phi: \mathcal{A} \longrightarrow H \\
& \phi\left(z_{i}+T_{2}\right)=f_{i} \\
& \phi\left(y_{i}+T_{2}\right)=e_{\lambda_{i}+1} e_{\lambda_{i}+2}+e_{\lambda_{i}+3} e_{\lambda_{i}+4}+\ldots+e_{\lambda_{i}+(2 p-3)} e_{\lambda_{i}+(2 p-2)},
\end{aligned}
$$

onde $\lambda_{i}=(i-1)(2 p-2)$. Note que basta definirmos $\phi$ nos geradores livres de $\mathcal{A}$, pois $H$ pertence a variedade de álgebras $\mathbb{Z}_{2}$-graduadas determinada por $T_{2}$ e $\mathcal{A}$ é a álgebra relativamente livre $\mathbb{Z}_{2}$-graduada dessa variedade.

Primeiro, para $m<p, \phi\left(y_{i}+T_{2}\right)^{m}$ é combinação linear de elementos da $K$-base de $H$ de comprimento $2 m$,

$$
e_{r_{1}} e_{r_{2}} \cdots e_{r_{2 m-1}} e_{r_{2 m}}
$$

onde $r_{j} \in\{\lambda+1, \lambda+2, \ldots, \lambda+(2 p-2)\}, r_{1}<\ldots<r_{2 m}$.

Suponha que $\phi\left(\sum \alpha_{s} u_{s}+T_{2}\right)=0$, onde

$$
u_{s}+T_{2}=y_{i_{s 1}}^{m_{s 1}} \cdots y_{i_{s k}}^{m_{s k}} z_{j_{s 1}} \cdots z_{j_{s l}}+T_{2}
$$

são monômios no conjunto (3.2) e $\alpha_{s} \in K$. Assim

$$
\begin{aligned}
\phi\left(u_{s}+T_{2}\right) & =\left(\sum_{r(1)} \alpha_{r 1} w_{i_{r(1)}}\right) \cdots\left(\sum_{r(k)} \alpha_{r(k)} w_{i_{r(k)}}\right) f_{j_{s 1}} \cdots f_{j_{s l}} \\
& =\sum \alpha_{(r(1), \ldots, r(k))} w_{i_{r(1)}} \cdots w_{i_{r(k)}} f_{j_{s_{1}}} \cdots f_{j_{s_{l}}}
\end{aligned}
$$

onde $w_{i_{r(j)}}$ são monômios da $K$-base de $H$ em $e_{\lambda_{i_{s j}}+1}, e_{\lambda_{i_{s j}}+2}, \ldots, e_{\lambda_{i_{s j}}+(2 p-3)}, e_{\lambda_{i_{s j}}+(2 p-2)}$, de comprimento $2 m_{s j}$, com $\lambda_{i_{s j}}=\left(i_{s j}-1\right)(2 p-2)$. Logo os elementos $w_{i_{r(1)}} \cdots w_{i_{r(k)}} f_{j_{s_{1}}} \cdots f_{j_{s_{l}}}$ estão na base linear de $H$, pois cada $w_{r(j)}$ depende dos índices $i_{s j}$, que estão ordenados. Além disso, cada $w_{r j}$ também depende do expoente $m_{s j}$. Com isso temos que os monômios $w_{i_{r(1)}} \cdots w_{i_{r(k)}} f_{j_{s_{1}}} \cdots f_{j_{s_{l}}}$ são unicamente determinados por $u_{s}+T_{2}$, isto é, eles são linearmente independentes em $H$.

$$
\begin{aligned}
\phi\left(\sum \alpha_{s} u_{s}+T_{2}\right) & =\sum \alpha_{s}\left(\sum \alpha_{(r(1), \ldots, r(k))} w_{i_{r(1)}} \cdots w_{i_{r(k)}} f_{j_{s_{1}}} \cdots f_{j_{s_{l}}}\right) \\
& =\sum \alpha_{s} \alpha_{(r(1), \ldots, r(k))} w_{i_{r(1)}} \cdots w_{i_{r(k)}} f_{j_{s_{1}}} \cdots f_{j_{s_{l}}} \\
& =0
\end{aligned}
$$


implica que $\alpha_{s}=0$, pois os $w_{i_{r(1)}} \cdots w_{i_{r(k)}} f_{j_{s_{1}}} \cdots f_{j_{s_{l}}}$ são distintos e pertencem a base de $H$, e os $\alpha_{(r(1), \ldots, r(k))} \neq 0$. Logo, $\phi$ é injetora.

Os seguintes resultados provavelmente são bem conhecidos quando $K$ é um corpo, mas não conseguimos encontrar as referências.

Corolário 3.1. A é um $K$-módulo livre com uma $K$-base formada pelos monômios

$$
y_{i_{1}}^{m_{1}} \cdots y_{i_{k}}^{m_{k}} z_{j_{1}} \cdots z_{j_{l}}+T_{2}
$$

onde $i_{1}<\ldots<i_{k}, j_{1}<\ldots<j_{l} ; 0<m_{1}, \ldots, m_{k}<p ; k, l \geq 0$ e $k+l>0$.

Demonstração. Pelo teorema anterior, as imagens dos monômios $y_{i_{1}}^{m_{1}} \cdots y_{i_{k}}^{m_{k}} z_{j_{1}} \cdots z_{j_{l}}+T_{2}$ pela $\phi$ são linearmente independentes sobre $K$ em $H$. Como eles são geradores de $\mathcal{A}$, eles são linearmente independentes sobre $K \mathrm{em} \mathcal{A}$.

Corolário 3.2. As identidades (3.1) formam uma conjunto gerador para o $T_{2}$-ideal das identidades de $H$, isto é, $T_{2}(H)=T_{2}$.

Demonstração. Como $\mathcal{A} \subseteq H$ pelo teorema anterior, temos que $T_{2}(H) \subseteq T_{2}(\mathcal{A})=T_{2}$. Mas $H$ satisfaz (3.1), logo $T_{2} \subseteq T_{2}(H)$. Assim, $T=T_{2}(H)$.

\subsection{Identidades de $H$}

Nessa seção, usaremos o Teorema 1.4 para encontrar uma base para as identidades ordinárias de $H$ e uma $K$-base para o quociente $K\langle X\rangle / T(H)$. O $T$-ideal das identidades de $H$ já foi descrito em [8] por Chiripov e Siderov quando $K$ é um corpo.

Seja $V$ o $T$-ideal de $K\langle X\rangle$ gerado pelos polinômios $\left[x_{1}, x_{2}, x_{3}\right]$ e $x^{p}$. O seguinte lema é bem conhecido quando $K$ é um corpo. Mas o lema continua verdadeiro mesmo quando $K$ é domínio de integridade, e a demonstração permanece a mesma (ver, por exemplo, [10]).

Lema 3.3. Seja $K$ um dominio de integridade de característica $p>2$. Para todos $g_{1}, g_{2}, g_{3}, g_{4} \in K\langle X\rangle$, temos o seguinte:

i) Os elementos $\left[g_{1}, g_{2}\right]+V$ são centrais em $K\langle X\rangle / V$. 
ii) $\left[g_{1}, g_{2}\right]\left[g_{3}, g_{4}\right]+V=-\left[g_{1}, g_{3}\right]\left[g_{2}, g_{4}\right]+V$.

iii) $\left[g_{1}, g_{2}\right]\left[g_{2}, g_{3}\right]+V=V$.

Usando esse lema, podemos encontrar um conjunto gerador para $K\langle X\rangle / V$.

Proposição 3.4. Um conjunto gerador de $K\langle X\rangle / V$ visto como $K$-módulo é

$$
x_{i_{1}}^{m_{1}} \cdots x_{i_{k}}^{m_{k}}\left[x_{j_{1}}, x_{j_{2}}\right] \cdots\left[x_{j_{2 l-1}}, x_{j_{2 l}}\right]+V \text {, }
$$

onde $k \geq 0, l \geq 0, k+l>0, i_{1}<\ldots<i_{k}, j_{1}<\ldots<j_{k}, 0<m_{i}<p$.

Demonstração. De fato, uma base de $K\langle X\rangle$ é composta por todos os monômios

$$
x_{i_{1}} x_{i_{2}} \cdots x_{i_{k}}
$$

onde $i_{1}, \ldots, i_{k}$ são inteiros positivos. Como $\left[x_{i_{1}}, x_{i_{2}}\right]+V$ é central em $K\langle X\rangle$,

$$
\begin{aligned}
x_{i_{1}} x_{i_{2}} \cdots x_{i_{k}}+V & =\left(x_{i_{2}} x_{i_{1}}+\left[x_{i_{1}}, x_{i_{2}}\right]\right) x_{i_{3}} \cdots x_{i_{k}}+V \\
& =x_{i_{2}} x_{i_{1}} x_{i_{3}} \cdots x_{i_{k}}+\left[x_{i_{1}}, x_{i_{2}}\right] x_{i_{3}} \cdots x_{i_{k}}+V \\
& =x_{i_{2}} x_{i_{1}} x_{i_{3}} \cdots x_{i_{k}}+x_{i_{3}} \cdots x_{i_{k}}\left[x_{i_{1}}, x_{i_{2}}\right]+V
\end{aligned}
$$

Usando esse procedimento várias vezes e o lema 3.3, podemos escrever cada monômio $x_{i_{1}} x_{i_{2}} \cdots x_{i_{k}}+V$ como combinação linear de polinômios do tipo

$$
x_{i_{1}^{\prime}}^{m_{1}} \cdots x_{i_{k}^{\prime}}^{m_{k}}\left[x_{j_{1}}, x_{j_{2}}\right] \cdots\left[x_{j_{2 l-1}}, x_{j_{2 l}}\right]+V
$$

onde $i_{1}^{\prime}<\ldots<i_{k}^{\prime}$. A seguir, usando os itens $(i i)$ e (iii) do lema (3.3), podemos ordenar os índices da parte comutador

$$
\left[x_{j_{1}}, x_{j_{2}}\right] \cdots\left[x_{j_{2 l-1}}, x_{j_{2 l}}\right]+V
$$

de modo que $j_{1}<\ldots<j_{2 l}$. Assim podemos escrever o monômio $x_{i_{1}} x_{i_{2}} \cdots x_{i_{k}}+V$ como uma combinação linear de polinômios

$$
x_{i_{1}^{\prime}}^{m_{1}} \cdots x_{i_{k}^{\prime}}^{m_{k}}\left[x_{j_{1}}, x_{j_{2}}\right] \cdots\left[x_{j_{2 l-1}}, x_{j_{2 l}}\right]+V
$$

onde $i_{1}^{\prime}<\ldots<i_{k}^{\prime}, j_{1}<\ldots<j_{2 l}, m_{1}, \ldots, m_{k}<p, k+l>0, k, l \geq 0$. 
Agora seja $\mathcal{D}$ a subálgebra de $\mathcal{A}$ gerada por $x_{i}+T_{2}(H)$, onde $x_{i}=y_{i}+z_{i}$. Como já vimos, $D$ é a álgebra relativamente livre da variedade determinada por $H$. Como isso temos o teorema (ver introdução).

Teorema 1.1 A álgebra $\mathcal{D}$ pode ser mergulhada em $H$, isto é, $\mathcal{D} \subset H$.

Demonstração. De fato $\mathcal{D} \simeq K\langle X\rangle / T(H)$ e $\mathcal{D} \subset K\langle Y, Z\rangle / T_{2}(H) \subset H$.

Mostraremos que $\mathcal{D}$ tem uma base semelhante ao conjunto gerador de $K\langle X\rangle / V$.

Proposição 3.5. Os elementos de $\mathcal{D}$ da forma

$$
x_{i_{1}}^{m_{1}} \cdots x_{i_{k}}^{m_{k}}\left[x_{j_{1}}, x_{j_{2}}\right] \cdots\left[x_{j_{2 l-1}}, x_{j_{2 l}}\right]+T_{2},
$$

onde $k \geq 0, l \geq 0, k+l>0, i_{1}<\ldots<i_{k}, j_{i}<\ldots<j_{k}, 0<m_{i}<p$, são linearmente independentes em $\mathcal{D}$.

Demonstração. Primeiro, note que como a característica de $K$ é $p$ e $y_{i}+T_{2}$ e $z_{i}+T_{2}$ comutam em $\mathcal{A}$, temos que $x_{i}^{p}+T_{2}=\left(y_{i}+z_{i}\right)^{p}+T_{2}=y_{i}^{p}+z_{i}^{p}+T_{2}=0$. Também, para $m<p, x_{i}^{m}+T_{2}=y_{i}^{m}+m y_{i}^{m-1} z_{i}+T_{2} \neq 0$ em $\mathcal{A}$. Assim,

$$
\begin{aligned}
{\left[x_{1}, x_{2}\right]+T_{2} } & =\left[y_{1}+z_{1}, y_{2}+z_{2}\right]+T_{2} \\
& =\left[y_{1}, y_{2}\right]+\left[y_{1}, z_{2}\right]+\left[z_{1}, y_{2}\right]+\left[z_{1}, z_{2}\right]+T_{2} \\
& =2 z_{1} z_{2}+T_{2} .
\end{aligned}
$$

Logo,

$$
x_{i_{1}}^{m_{1}} \cdots x_{i_{k}}^{m_{k}}\left[x_{j_{1}}, x_{j_{2}}\right] \cdots\left[x_{j_{2 l-1}}, x_{j_{2 l}}\right]+T_{2}=2^{l} y_{i_{1}}^{m_{1}} \cdots y_{i_{k}}^{m_{k}} z_{j_{1}} \cdots z_{j_{2 l}}+\cdots+T_{2} .
$$

Note que $y_{i_{1}}^{m_{1}} \cdots y_{i_{k}}^{m_{k}} z_{j_{1}} \cdots z_{j_{2 l}}$ tem grau total nas varáveis $Y$ igual a $m_{1}+\ldots+m_{k}$ e todos os outros monômios na soma do segundo membro da equação tem grau total nas variáveis $Y$ menor que $m_{1}+\ldots+m_{k}$. Chamaremos $y_{i_{1}}^{m_{1}} \cdots y_{i_{k}}^{m_{k}} z_{j_{1}} \cdots z_{j_{2 l}}+T_{2}$ de monômio líder. Note que dois elementos distintos do tipo (3.3) tem monômios líderes distintos.

Se tivermos uma combinação linear de elementos (3.3), no conjunto dos monômios líderes $y_{i_{1}}^{m_{1}} \cdots y_{i_{k}}^{m_{k}} z_{j_{1}} \cdots z_{j_{2 l}}+T_{2}$ que aparecem nessa combinação linear, considere a seguinte ordem: $u_{1}+T_{2}>u_{2}+T_{2}$ se no monômio $u_{1}$, o grau total nas variáveis $Y$ é maior que o grau total nas variáveis $Y$ do monômio $u_{2}$. Se o grau total nas variáveis $Y$ de $u_{1}$ e 
$u_{2}$ são iguais, $u_{1}+T_{2}>u_{2}+T_{2}$ se o grau total nas variáveis $Z$ de $u_{1}$ é maior que o grau total nas variáveis $Z$ de $u_{2}$. Por exemplo, $y_{1}^{2} y_{2}+T_{2}>y_{3} y_{4}+T_{2}$ e $y_{1}^{2} y_{3} z_{1}+T_{2}<y_{1}^{2} y_{2}+T_{2}$.

Como os monômios $u=y_{i_{1}}^{m_{1}} \cdots y_{i_{k}}^{m_{k}} z_{j_{1}} \cdots z_{j_{2 l}}+T_{2}$ são linearmente independentes em $\mathcal{A}$ e não podem ser combinação linear de monômios $u^{\prime}+T_{2} \operatorname{com} u^{\prime}<u$, temos que os coeficientes que multiplicam esses monômios devem ser zero. Logo, os elementos (3.3) são linearmente independentes.

Proposição 3.6. A álgebra $\mathcal{D}$ satisfaz as identidades $\left[x_{1}, x_{2}, x_{3}\right]$ e $x_{1}^{p}$.

Demonstração. Seja $f\left(x_{1}, \ldots, x_{n}\right)+T_{2} \in \mathcal{D}$. Como $x_{i}=y_{i}+z_{i}$, podemos escrever $f+T_{2}=$ $f^{(0)}+f^{(1)}+T_{2}$, onde $f^{(0)}$ pertence a parte par e $f^{(1)}$ pertence a parte ímpar de $K\langle Y, Z\rangle$. Seja $f_{1}+T_{2}, f_{2}+T_{2}, f_{3}+T_{2} \in \mathcal{D}$.

$$
\begin{aligned}
{\left[f_{1}, f_{2}, f_{3}\right]+T_{2} } & =\left[f_{1}^{(0)}+f_{1}^{(1)}, f_{2}^{(0)}+f_{2}^{(1)}, f_{3}\right]+T_{2} \\
& =\left[2 f_{1}^{(1)} f_{2}^{(1)}, f_{3}\right]+T_{2} \\
& =0
\end{aligned}
$$

pois $f_{1}^{(1)} f_{2}^{(1)}$ pertence a parte par de $K\langle Y, Z\rangle / T_{2}$. Também,

$$
\begin{aligned}
f_{1}^{p}+T_{2} & =\left(f_{1}^{(0)}+f_{1}^{(1)}\right)^{p}+T_{2} \\
& =\left(f_{1}^{(0)}\right)^{p}+\left(f_{1}^{(1)}\right)^{p}+T_{2} \\
& =0 .
\end{aligned}
$$

Assim, pelas proposições 3.5 e 3.4, concluímos que (3.3) formam uma base de $\mathcal{D}$.

Considere agora o seguinte homomorfismo,

$$
\begin{aligned}
& K\langle X\rangle \longrightarrow \mathcal{D} \\
& x_{i} \mapsto x_{i}+T_{2},
\end{aligned}
$$

isto é, $f\left(x_{1}, \ldots, x_{n}\right) \mapsto f\left(x_{1}, \ldots, x_{n}\right)+T_{2}$. Pela proposição $3.6, V$ está contido no núcleo desse homomorfismo. Logo, o homomorfismo

$$
\begin{aligned}
& \Psi: K\langle X\rangle / V \longrightarrow \mathcal{D} \\
& f\left(x_{1}, \ldots, x_{n}\right)+V \mapsto f\left(x_{1}, \ldots, x_{n}\right)+T_{2} .
\end{aligned}
$$


está bem definido.

Mas então,

$$
\begin{aligned}
& \Psi: K\langle X\rangle / V \longrightarrow \mathcal{D} \\
& x_{i_{1}}^{m_{1}} \cdots x_{i_{k}}^{m_{k}}\left[x_{j_{1}}, x_{j_{2}}\right] \cdots\left[x_{j_{2 l-1}}, x_{j_{2 l}}\right]+V \mapsto x_{i_{1}}^{m_{1}} \cdots x_{i_{k}}^{m_{k}}\left[x_{j_{1}}, x_{j_{2}}\right] \cdots\left[x_{j_{2 l-1}}, x_{j_{2 l}}\right]+T_{2} .
\end{aligned}
$$

leva geradores de $K\langle X\rangle / V$ em base de $\mathcal{D}$. Portanto, $\Psi$ é um isomorfismo.

Com isso, temos os seguintes corolários do teorema (1.1).

Corolário 1.2 Seja $H$ a álgebra de Grassmann não-unitária de dimensão infinita sobre um dominio de integridade de característica $p>2$. O conjunto das identidades de $H$ é o $T$-ideal de $K\langle X\rangle$ gerado por $\left[x_{1}, x_{2}, x_{3}\right]$ e $x^{p}$.

Demonstração. De fato, $K\langle X\rangle / V \simeq \mathcal{D} \simeq K\langle X\rangle / T(H)$. Logo $T(H)=V$.

Obtemos então uma demonstração simples do teorema que já foi demonstrado por Chiripov e Siderov em [8] quando $K$ é um corpo.

Corolário $1.3 K\langle X\rangle / V$ é um $K$-módulo livre com uma $K$-base formada pelos polinômios

$$
x_{i_{1}}^{m_{1}} \cdots x_{i_{k}}^{m_{k}}\left[x_{j_{1}}, x_{j_{2}}\right] \cdots\left[x_{j_{2 l-1}}, x_{j_{2 l}}\right]+V
$$

ondek $\geq 0, l \geq 0, k+l>0, i_{1}<\ldots<i_{k}, j_{1}<\ldots<j_{k}, 0<m_{i}<p$.

\subsection{Polinômios Centrais de $H$}

Nessa seção encontraremos o $T$-subespaço de polinômios centrais de $H$. Usaremos vários resultados de [6]. Mostraremos também que o $T$-subespaço de polinômios centrais de $H$ não é finitamente gerado como $T$-subespaço.

Começamos observando que

Lema 3.7. V é um T-ideal multi-homogêneo.

Demonstração. De fato, como todo polinômio de $K\langle X\rangle / V$ pode ser escrito como combinação linear de elementos da forma (3.4), temos que $K\langle X\rangle / V$ é graduado pelos seus multi-graus. 
Com isso temos

Lema 1. O T-subespaço de polinômios centrais de $H, C(H)$, é multi-homogêneo.

Demonstração. Seja $f \in C(H)$. Decomponha $f=\sum f_{i}$ como soma de suas componentes multi-homogêneas. Então

$$
\left[f, x_{1}\right]=\sum\left[f_{i}, x_{1}\right] \in T(H) .
$$

Como as componentes multi-homogêneas de $\left[f, x_{1}\right]$ são $\left[f_{i}, x_{1}\right]$, temos que $\left[f_{i}, x_{1}\right] \in T(H)$. Assim $f_{i} \in C(H)$.

Os lemas 3.8, 3.9, 3.10 e o teorema 3.13 que apresentaremos a seguir podem ser encontrados em [6]. Embora estamos considerando $K$ um domínio de integridade de característica $p>2$, as demonstrações são as mesmas.

Lema 3.8. Seja $K$ um dominio de integridade de característica $p>2$ e seja $g=$ $g\left(x_{3}, \ldots, x_{l}\right) \in K\langle X\rangle$ um polinômio que não depende de $x_{2}$. Suponha que $x_{2} g+V$ é central em $K\langle X\rangle / V$. Então $g \in V$.

Demonstração. Primeiro, como $x_{2} g+V$ é central em $K\langle X\rangle / V$, temos que $0=\left[x_{1}, x_{2} g\right]+$ $V=x_{2}\left[x_{1}, g\right]+\left[x_{1}, x_{2}\right] g+V$. Assim,

$$
x_{2}\left[x_{1}, g\right]+V=-\left[x_{1}, x_{2}\right] g+V .
$$

Suponha que $g+V=\sum_{t} \beta_{t} b_{t}$, onde $\beta \in K$ e $b_{t}$ são elementos distintos da forma (3.4). Como $g=g\left(x_{3}, \ldots, x_{l}\right)$ não depende de $x_{1}$ e $x_{2}$, temos que os elementos $b_{t}$ também não dependem. Para cada $t$,

$$
\left[x_{1}, b_{t}\right]+V=\sum_{k} \alpha_{k}^{(t)} c_{k}^{(t)}
$$

onde $\alpha_{t} \in K$ e $c_{k}^{(t)}$ são elementos da forma (3.4) que não dependem de $x_{2}$. Pelo lema 3.3, podemos assumir que $j_{1}=1$ na expressão (3.4). Segue que

$$
x_{2}\left[x_{1}, g\right]+V=\sum_{t} \sum_{k} \alpha_{k}^{(t)} x_{2} c_{k}^{(t)} .
$$


Note que para cada $t$ e $k, x_{2} c_{k}^{(t)}$ é um elemento da forma (3.4) com $i_{1}=2$ e $2 \notin\left\{j_{1}, \ldots, j_{2 l}\right\}$. Logo, $x_{2}\left[x_{1}, g\right]+V$ é combinação linear de elementos da forma (3.4),

$$
x_{i_{1}}^{m_{1}} \cdots x_{i_{k}}^{m_{k}}\left[x_{j_{1}}, x_{j_{2}}\right] \cdots\left[x_{j_{2 l-1}}, x_{j_{2 l}}\right]+V
$$

tal que $x_{2}$ está contido na parte não-comutador $x_{i_{1}}^{m_{1}} \cdots x_{i_{k}}^{m_{k}}$ e a parte comutador

$$
\left[x_{j_{1}}, x_{j_{2}}\right] \cdots\left[x_{j_{2 l-1}}, x_{j_{2 l}}\right]
$$

não depende de $x_{2}$.

Por outro lado, $\left[x_{1}, x_{2}\right] g+V=\sum_{t} \beta_{t}\left[x_{1}, x_{2}\right] b_{t}$. Como os elementos $b_{t}$ não dependem de $x_{1}$ e $x_{2}$, e $\left[x_{1}, x_{2}\right]+V$ é central em $K\langle X\rangle / V$, os produtos $\left[x_{1}, x_{2}\right] b_{t}$ são elementos distintos da forma (3.4). Logo, $\left[x_{1}, x_{2}\right] g+V$ é uma combinação linear de elementos da forma (3.4) com $j_{2}=2$ e $2 \notin\left\{i_{1}, \ldots, i_{2 k}\right\}$. Em outras palavras, $\left[x_{1}, x_{2}\right] g+V$ é uma combinação linear de elementos da forma (3.4) com $x_{2}$ contido na parte comutador do produto e a parte não-comutador não depende de $x_{2}$. Mas os elementos da forma (3.4) formam uma base de $K\langle X\rangle / V$, assim devemos ter

$$
x_{2}\left[x_{1}, g+V\right]=-\left[x_{1}, x_{2}\right] g+V=0 .
$$

Portanto, $\sum_{t} \beta_{t}\left[x_{1}, x_{2}\right] b_{t}=\left[x_{1}, x_{2}\right] g+V=0$. Assim, $\beta_{t}=0$ para todo $t$ e $g+V=$ $\sum_{t} \beta_{t} b_{t}=0$, isto é, $g \in V$.

Lema 3.9. Seja K um dominio de integridade de característica $p>2$. Suponha que $f=f\left(x_{2}, \ldots, x_{l}\right) \in K\langle X\rangle$ é um polinômio homogêneo de grau 1 em $x_{2}$ e que $f+V$ é central em $K\langle X\rangle / V$. Então $f+V$ pertence ao $T$-espaço de $K\langle X\rangle / V$ gerado por $\left[x_{1}, x_{2}\right]+V$.

Demonstração. Seja $f=\sum_{i} \alpha_{i} a_{i} x_{2} b_{i}$ onde $\alpha_{i} \in K$ e $a_{i}, b_{i}$ são monômios. Como $a x_{2} b=$ $x_{2} a b+\left[a, x_{2} b\right]$, temos

$$
f=x_{2} g\left(x_{3}, \ldots, x_{l}\right)+h\left(x_{1}, \ldots, x_{l}\right)
$$

onde $h\left(x_{1}, \ldots, x_{l}\right)=\sum_{i} \alpha_{i}\left[a_{i}, x_{2} b_{i}\right]$ pertence ao $T$-espaço gerado por $\left[x_{1}, x_{2}\right]$ e $g=g\left(x_{3}, \ldots, x_{l}\right)$ não depende de $x_{2}$. Mas como $f+V$ e $h+V$ são centrais em $K\langle X\rangle / V$, temos que $x_{2} g+V$ também é central. Pelo lema anterior, $g \in V$. Segue que

$$
f+V=h+V
$$


Assim, $f+V$ pertence ao $T$-espaço de $K\langle X\rangle / V$ gerado por $\left[x_{1}, x_{2}\right]+V$.

Lema 3.10. Suponha que char $(K)=p>2$ e seja $f\left(x_{2}, \ldots, x_{l}\right) \in K\langle X\rangle$ um polinômio homogêneo de grau $m$ em $x_{2}, 0<m<p$. Suponha que $f+V$ é central em $K\langle X\rangle / V$. Então $f+V$ pertence ao $T$-espaço de $K\langle X\rangle / V$ gerado por $\left[x_{1}, x_{2}\right]+V$.

Demonstração. Seja $h=h\left(x_{1}^{\prime}, \ldots, x_{m}^{\prime}, x_{3}, \ldots, x_{l}\right)$ a componente homogênea de $f\left(x_{1}^{\prime}+\cdots+x_{m}^{\prime}, x_{3}, \ldots, x_{l}\right)$ que é multilinear com relação a $x_{1}^{\prime}, \ldots, x_{m}^{\prime}$. Como $f+V$ é central em $K\langle X\rangle / V$, assim é $h+V$. Também, como

$$
h\left(x_{2}, \ldots, x_{2}, x_{3}, \ldots, x_{l}\right)=m ! f\left(x_{2}, x_{3}, \ldots, x_{l}\right),
$$

é suficiente mostrar que $h+V$ pertence ao $T$-espaço de $K\langle X\rangle / V$ gerado por $\left[x_{1}, x_{2}\right]+V$. Mas

$$
h=h\left(x_{1}, \ldots, x_{m}, x_{m+1}, \ldots, x_{m+l}\right)
$$

é homogêneo de grau 1 em $x_{2}$. O resultado segue pelo lema anterior.

Agora seja $C$ o $K$-submódulo de $K\langle X\rangle$ gerado por $\left[g_{1}, g_{2}\right]$, onde $g_{1}, g_{2}$ são monômios. Temos os seguintes lemas.

Lema 3.11. $C$ é gerado como $K$-módulo por todos os comutadores $[x, g]$, onde $g$ é um monômio de $K\langle X\rangle$ e $x \in X$.

Demonstração. Seja $C^{\prime}$ o $K$-submódulo de $K\langle X\rangle$ gerado por todos os comutadores $[x, g]$, onde $g$ é um monômio de $K\langle X\rangle$ e $x \in X$. Então $C^{\prime} \subseteq C$. Para provar a inclusão $C \subseteq C^{\prime}$, considere a seguinte igualdade em $K\langle X\rangle$ :

$$
[a x, b]+[x b, a]+[b a, x]=0
$$

onde $a, b \in K\langle X\rangle$ e $x \in X$. Logo,

$$
[a x, b]+C^{\prime}=[a, x b]+C^{\prime}
$$


Sejam $a, b$ monômios de $K\langle X\rangle$ onde $a=x_{i_{1}} \cdots x_{i_{k}}, x_{i} \in X$. Então

$$
\begin{aligned}
{[a, b]+C^{\prime} } & =\left[x_{i_{1}} \cdots x_{i_{k}}, b\right]+C^{\prime} \\
& =\left[x_{i_{1}} \cdots x_{i_{k-1}}, x_{i_{k}} b\right]+C^{\prime} \\
& =\quad \vdots \\
& =\left[x_{i_{1}}, x_{i_{2}} \cdots x_{i_{k}} b\right]+C^{\prime} \\
& =C^{\prime} .
\end{aligned}
$$

Portanto $[a, b] \in C^{\prime}$, ou seja, $C \subseteq C^{\prime}$.

Lema 3.12. Seja $\left[x_{i_{1}}, g\right] \in K\langle X\rangle$ onde $x_{i_{1}} \in X$ e $g=x_{j_{1}} \cdots x_{j_{k}}$ é um monômio de $K\langle X\rangle$. Se $\left[x_{i_{1}}, g\right]$ tem grau $p$ em relação a todas as variáveis $x_{i_{1}}, x_{j_{1}}, \ldots, x_{j_{k}}$, então $\left[x_{i_{1}}, g\right] \in V$.

Demonstração. Usando que $[a b, c]=a[b, c]+[a, c] b$, onde $a, b, c \in K\langle X\rangle$ temos

$$
\left[x_{i_{1}}, x_{j_{1}} \cdots x_{j_{k}}\right]=\sum_{s=1}^{k} x_{j_{1}} \cdots x_{j_{s-1}}\left[x_{i_{1}}, x_{j_{s}}\right] x_{j_{s+1}} \cdots x_{j_{k}} .
$$

Se $j_{s}=i_{1}$ então $\left[x_{1_{1}}, x_{j_{s}}\right]=0$. Como o grau de $x_{j_{1}}$ é $p$, suponha que $j_{s_{1}}, \ldots, j_{s_{p}}=j_{1}$. Em

$$
\sum_{s=1}^{k} x_{j_{1}} \cdots x_{j_{s-1}}\left[x_{i_{1}}, x_{j_{s}}\right] x_{j_{s+1}} \cdots x_{j_{k}} .
$$

vamos tomar apenas as parcelas onde $\left[x_{i_{1}}, x_{j_{1}}\right]$ aparece, isto é,

$$
\sum_{r=1}^{p} x_{j_{1}} \cdots x_{j_{s_{r}-1}}\left[x_{i_{1}}, x_{j_{1}}\right] x_{j_{s_{r}+1}} \cdots x_{j_{k}}
$$

Temos então que, módulo $V$,

$$
\begin{aligned}
\sum_{r=1}^{p} x_{j_{1}} \cdots x_{j_{s_{r}-1}}\left[x_{i_{1}}, x_{j_{1}}\right] x_{j_{s_{r}+1}} \cdots x_{j_{k}}+V & =p g^{\prime} x_{j_{2}}^{p-1}\left[x_{i_{1}}, x_{j_{1}}\right]+V \\
& =0
\end{aligned}
$$

onde $g^{\prime}$ é obtido de $g$ por exclusão de todas as variáveis $x_{j_{1}}$. Podemos fazer isso pois temos 
a igualdade

$$
\begin{aligned}
x_{1} x_{2}\left[x_{1}, x_{3}\right]+V & =\left(x_{2} x_{1}+\left[x_{1}, x_{2}\right]\right)\left[x_{1}, x_{3}\right]+V \\
& =x_{2} x_{1}\left[x_{1}, x_{3}\right]+\left[x_{1}, x_{2}\right]\left[x_{1}, x_{3}\right]+V \\
& =x_{2} x_{1}\left[x_{1}, x_{3}\right]+V
\end{aligned}
$$

isto é, se uma variável aparece na parte comutador e na parte não comutador, então essa variável comuta com qualquer outra, módulo $V$.

Como as variáveis $x_{j_{2}}, \ldots, x_{j_{k}}$ também têm grau $p$, podemos fazer o mesmo considerando as parcelas de $\left[x_{i_{1}}, g\right]$ onde $\left[x_{i_{1}}, x_{j_{2}}\right]$ aparece e assim por diante. Assim, $\left[x_{i_{1}}, g\right] \in$ $V$.

Teorema 3.13. O K-módulo $C(H)$ é gerado, módulo $V$, por

$$
\begin{aligned}
& {\left[g_{1}, g_{2}\right]+V} \\
& x_{1}^{p-1}\left[x_{1}, x_{2}\right] x_{2}^{p-1} \cdots x_{2 k-1}^{p-1}\left[x_{2 k-1}, x_{2 k}\right] x_{2 k}^{p-1}+V
\end{aligned}
$$

onde $k=1,2, \ldots, g_{1}, g_{2}$ são monômios em $K\langle X\rangle$ e $x_{i} \in X$ para cada $i$.

Demonstração. Seja $f=f\left(x_{1}, \ldots, x_{l}\right) \in C(H)$ um polinômio multi-homogêneo de grau $m_{i}$ em $x_{i}, i=1, \ldots, l$. Então, $f+V$ é central em $K\langle X\rangle / V$. Suponha que em $f, 0<m_{i}<p$ para algum $i$. Sem perda de generalidade, podemos supor que $i=1$. Logo, pelo lema anterior, $f+V$ pertence ao $T$-subespaço de $K\langle X\rangle / V$ gerado por $\left[x_{1}, x_{2}\right]+V$.

Agora suponha que $m_{i}=p$ para todo $i$. Então pela proposição (3.4), $f+V$ é uma combinação linear de elementos da forma (3.4). Mas todos os elementos da forma (3.4) com $m_{i}=p$ para todo $i$ são do tipo

$$
x_{1}^{p-1}\left[x_{1}, x_{2}\right] x_{2}^{p-1} \cdots x_{2 k-1}^{p-1}\left[x_{2 k-1}, x_{2 k}\right] x_{2 k}^{p-1}+V,
$$

$\operatorname{com} k=1,2, \ldots$

Note que pelo lema anterior, os polinômios

$$
x_{1}^{p-1}\left[x_{1}, x_{2}\right] x_{2}^{p-1} \cdots x_{2 k-1}^{p-1}\left[x_{2 k-1}, x_{2 k}\right] x_{2 k}^{p-1},
$$

não pertencem à $C$, pois tem grau $p$ em relação a todas as variáveis. 
Note que pelos lemas e teorema anterior temos que

$$
C(H) / V=(C+V) / V \oplus \bigoplus_{k=1}^{\infty}\left(C_{k}+V\right) / V
$$

onde $C$ é o $K$-submódulo gerado por $\left[g_{1}, g_{2}\right]$, onde $g_{1}, g_{2}$ são monômios, e $C_{k}$ é o $K$ subespaço gerado por $x_{1}^{p-1}\left[x_{1}, x_{2}\right] x_{2}^{p-1} \cdots x_{2 k-1}^{p-1}\left[x_{2 k-1}, x_{2 k}\right] x_{2 k}^{p-1}\left(x_{i} \in X\right)$.

Agora demonstraremos que $C(H)$ não é finitamente gerado como um $T$-espaço. Começaremos com alguns lemas.

Lema 3.14. Seja $h\left(x_{1}, x_{2}, x_{3}\right)=\left(x_{1}+x_{2}\right)^{p-1}\left[x_{1}+x_{2}, x_{3}\right] x_{3}^{p-1}$, onde $x_{1}, x_{2}, x_{3} \in X$. Ent $\tilde{a} O$

$$
h+V=x_{1}^{p-1}\left[x_{1}, x_{3}\right] x_{3}^{p-1}+x_{2}^{p-1}\left[x_{2}, x_{3}\right] x_{3}^{p-1}+f+V,
$$

onde $f+V$ pertence ao $T$-subespaço de $K\langle X\rangle / V$ gerado por $\left[x_{1}, x_{2}\right]+V$.

Demonstração. Seja $g\left(x_{1}, x_{2}\right)=\left(x_{1}+x_{2}\right)^{p-1}$. Então,

$$
g\left(x_{1}, x_{2}\right)=x_{1}^{p-1}+g_{(p-2,1)}+g_{(p-3,2)}+\ldots+g_{(1, p-2)}+x_{2}^{p-1},
$$

onde $g_{(i, j)}$ é um polinômio em $x_{1}, x_{2}$ com multi-grau $(i, j)$. Logo

$$
\begin{aligned}
h\left(x_{1}, x_{2}, x_{3}\right) & =g\left(x_{1}, x_{2}\right)\left[x_{1}, x_{3}\right] x_{3}^{p-1}+g\left(x_{1}, x_{2}\right)\left[x_{2}, x_{3}\right] x_{3}^{p-1} \\
& =x_{1}^{p-1}\left[x_{1}, x_{3}\right] x_{3}^{p-1}+x_{2}^{p-1}\left[x_{2}, x_{3}\right] x_{3}^{p-1}+f\left(x_{1}, x_{2}, x_{3}\right),
\end{aligned}
$$

onde

$$
\begin{aligned}
f\left(x_{1}, x_{2}, x_{3}\right)= & \left(g_{(p-2,1)}+g_{(p-3,2)}+\ldots+g_{(1, p-2)}+x_{2}^{p-1}\right)\left[x_{1}, x_{3}\right] x_{3}^{p-1} \\
& +\left(x_{1}^{p-1}+g_{(p-2,1)}+g_{(p-3,2)}+\ldots+g_{(1, p-2)}\right)\left[x_{2}, x_{3}\right] x_{3}^{p-1} .
\end{aligned}
$$

Como $h\left(x_{1}, x_{2}, x_{3}\right)+V, x_{1}^{p-1}\left[x_{1}, x_{3}\right] x_{3}^{p-1}+V, x_{2}^{p-1}\left[x_{2}, x_{3}\right] x_{3}^{p-1}+V$ são centrais em $K\langle X\rangle / V$, temos que $f\left(x_{1}, x_{2}, x_{3}\right)+V$ é central. Mas pelo corolário ??, todas as componentes multi-homogêneas de $f\left(x_{1}, x_{2}, x_{3}\right)+V$ são centrais em $K\langle X\rangle / V$. Mas todas as componentes multi-homogêneas de $f\left(x_{1}, x_{2}, x_{3}\right)+V$ tem grau $m$ em $x_{2}$ onde $0<m<p$. Portanto, pelo lema 3.10, todas essas componentes pertencem ao $T$-espaço gerado por $\left[x_{1}, x_{2}\right]+V$. Assim $f\left(x_{1}, x_{2}, x_{3}\right)+V$ pertence ao $T$-espaço gerado por $\left[x_{1}, x_{2}\right]+V$ em $K\langle X\rangle / V$. 
Analogamente, temos que

$$
x_{1}^{p-1}\left[x_{1}, x_{2}+x_{3}\right]\left(x_{2}+x_{3}\right)^{p-1}+V=x_{1}^{p-1}\left[x_{1}, x_{2}\right] x_{2}^{p-1}+x_{1}^{p-1}\left[x_{1}, x_{3}\right] x_{3}^{p-1}+f+V
$$

onde $f$ pertence ao $T$-espaço de $K\langle X\rangle / V$ gerado por $\left[x_{1}, x_{2}\right]+V$.

Pelo lema anterior,

$$
\left(x_{1}+x_{2}\right)^{p-1}\left[x_{1}+x_{2}, x_{3}\right] x_{3}^{p-1}-x_{1}^{p-1}\left[x_{1}, x_{3}\right] x_{3}^{p-1}-x_{2}^{p-1}\left[x_{2}, x_{3}\right] x_{3}^{p-1}-f \in V .
$$

Como $V$ é um $T$-ideal, para todos $f_{1}, f_{2}, f_{3}$ em $K\langle X\rangle$, fazendo a substituição $x_{1} \mapsto f_{1}, x_{2} \mapsto f_{2}, x_{3} \mapsto f_{3}$ temos

$$
\left(f_{1}+f_{2}\right)^{p-1}\left[f_{1}+f_{2}, f_{3}\right] f_{3}^{p-1}-f_{1}^{p-1}\left[f_{1}, f_{3}\right] f_{3}^{p-1}-f_{2}^{p-1}\left[f_{2}, f_{3}\right] f_{3}^{p-1}-f^{\prime} \in V .
$$

onde $f^{\prime}$ pertence ao $T$-espaço de $K\langle X\rangle / V$ gerado por $\left[x_{1}, x_{2}\right]+V$.

Logo, temos o seguinte corolário

Corolário 3.15. Sejam $f_{1}, f_{2}$ polinômios de $K\langle X\rangle$. Então,

$$
f_{1}^{p-1}\left[f_{1}, f_{2}\right] f_{2}^{p-1}+V=\sum_{i} \alpha_{i} u_{i_{1}}^{p-1}\left[u_{i_{1}}, u_{i_{2}}\right] u_{i_{2}}^{p-1}+f+V
$$

onde $u_{i_{j}}$ são monômios e $f$ pertence ao $T$-espaço de $K\langle X\rangle / V$ gerado por $\left[x_{1}, x_{2}\right]+V$.

Demonstração. Seja $f_{1}=\beta_{1} u_{1}+\beta_{2} u_{2} \in K\langle X\rangle$ onde $u_{1}$ e $u_{2}$ são monômios e $\beta_{1}, \beta_{2} \in K$. Pelo que foi dito acima,

$$
\begin{aligned}
f_{1}^{p-1}\left[f_{1}, f_{2}\right] f_{2}^{p-1}+V & =\left(\beta_{1} u_{1}+\beta_{2} u_{2}\right)^{p-1}\left[\beta_{1} u_{1}+\beta_{2} u_{2}, f_{2}\right] f_{2}^{p-1} \\
& =\left(\beta_{1} u_{1}\right)^{p-1}\left[\beta_{1} u_{1}, f_{2}\right] f_{2}^{p-1}+\left(\beta_{2} u_{2}\right)^{p-1}\left[\beta_{2} u_{2}, f_{2}\right] f_{2}^{p-1}+f+V \\
& =\beta_{1}^{p} u_{1}^{p-1}\left[u_{1}, f_{2}\right] f_{2}^{p-1}+\beta_{2}^{p} u_{2}^{p-1}\left[u_{2}, f_{2}\right] f_{2}^{p-1}+f+V,
\end{aligned}
$$

onde $f$ pertence ao $T$-espaço de $K\langle X\rangle / V$ gerado por $\left[x_{1}, x_{2}\right]+V$.

Por indução no número de parcelas de $f_{1}$ temos que se $f_{1}=\beta_{1} u_{1}+\ldots+\beta_{k} u_{k}$, onde $u_{1}, \ldots, u_{k}$ são monômios e $\beta_{1}, \ldots, \beta_{k} \in K$, então

$$
f_{1}^{p-1}\left[f_{1}, f_{2}\right] f_{2}^{p-1}+V=\beta_{1}^{p} u_{1}^{p-1}\left[u_{1}, f_{2}\right] f_{2}^{p-1}+\ldots \beta_{k}^{p} u_{k}^{p-1}\left[u_{k}, f_{2}\right] f_{2}^{p-1}+f+V,
$$


onde $f$ pertence ao $T$-espaço de $K\langle X\rangle / V$ gerado por $\left[x_{1}, x_{2}\right]+V$. Fazendo o mesmo para $f_{2}$, obtemos o resultado desejado.

Agora, mostraremos que os monômios $u_{i_{j}}$ que aparecem no corolário acima tem grau 1 , isto é, $u_{i_{j}} \in X$.

Lema 3.16. $h\left(x_{1}, x_{2}, x_{3}\right)=\left(x_{1} x_{2}\right)^{p-1}\left[x_{1} x_{2}, x_{3}\right] x_{3}^{p-1} \in V$ para todos $x_{1}, x_{2}, x_{3} \in X$.

Demonstração. Primeiro, como $\left[x_{1}, x_{2}\right]+V$ é central em $K\langle X\rangle / V$,

$$
\begin{aligned}
h+V & =\left(x_{1} x_{2}\right)^{p-1} x_{1}\left[x_{2}, x_{3}\right] x_{3}^{p-1}+\left(x_{1} x_{2}\right)^{p-1}\left[x_{1}, x_{3}\right] x_{2} x_{3}^{p-1}+V \\
& =\left(x_{1} x_{2}\right)^{p-1} x_{1}\left[x_{2}, x_{3}\right] x_{3}^{p-1}+\left(x_{1} x_{2}\right)^{p-1} x_{2}\left[x_{1}, x_{3}\right] x_{3}^{p-1}+V, \\
& =h_{1}+h_{2}+V
\end{aligned}
$$

onde $h_{1}=\left(x_{1} x_{2}\right)^{p-1} x_{1}\left[x_{2}, x_{3}\right] x_{3}^{p-1}$ e $h_{2}=\left(x_{1} x_{2}\right)^{p-1} x_{2}\left[x_{1}, x_{3}\right] x_{3}^{p-1}$.

Agora, como $\left[x_{1}, x_{2}\right]\left[x_{2}, x_{3}\right] \in V$, temos que

$$
\begin{aligned}
h_{1}+V & =\underbrace{x_{1} x_{2} \cdots x_{1} x_{2} \cdots x_{1} x_{2}}_{(p-1) \text { vezes }} x_{1}\left[x_{2}, x_{3}\right] x_{3}^{p-1}+V \\
& =x_{1} x_{2} \cdots\left(x_{2} x_{1}+\left[x_{1}, x_{2}\right]\right) \cdots x_{1} x_{2} x_{1}\left[x_{2}, x_{3}\right] x_{3}^{p-1}+V \\
& =x_{1} x_{2} \cdots x_{2} x_{1} \cdots x_{1} x_{2} x_{1}\left[x_{2}, x_{3}\right] x_{3}^{p-1}+\left(x_{1} x_{2}\right)^{p-2} x_{1}\left[x_{1}, x_{2}\right]\left[x_{2}, x_{3}\right] x_{3}^{p-1}+V \\
& =x_{1} x_{2} \cdots x_{2} x_{1} \cdots x_{1} x_{2} x_{1}\left[x_{2}, x_{3}\right] x_{3}^{p-1}+V \\
& \quad \vdots \\
& =x_{2}^{p-1} x_{1}^{p-1} x_{1}\left[x_{2}, x_{3}\right] x_{3}^{p-1}+V \\
& =x_{2}^{p-1} x_{1}^{p}\left[x_{2}, x_{3}\right] x_{3}^{p-1}+V \\
& =0
\end{aligned}
$$

pois $x_{1}^{p} \in V$. Logo $h_{1} \in V$. Analogamente,mostra-se que $h_{2} \in V$.

Usando o mesmo método, mostra-se também que $x_{1}^{p-1}\left[x_{1}, x_{2} x_{3}\right]\left(x_{2} x_{3}\right)^{p-1} \in V$ para todos $x_{1}, x_{2}, x_{3} \in X$. Logo, para todos os monômios $u_{1}$ e $u_{2}$ de $K\langle X\rangle$ (a menos dos monômios $u_{1}$ e $u_{2}$ de grau 1 ), temos que $u_{1}^{p-1}\left[u_{1}, u_{2}\right] u_{2}^{p-1} \in V$. De fato, se $u_{1}$ tem grau maior que 1 , então $u_{1}=u_{1}^{\prime} u_{1}^{\prime \prime}$, onde $u^{\prime}$ e $u_{1}^{\prime \prime}$ são monômios. Como $\left(x_{1} x_{2}\right)^{p-1}\left[x_{1} x_{2}, x_{3}\right] x_{3}^{p-1} \in$ $V$ e $V$ é um $T$-ideal, fazendo a substituição $x_{1} \mapsto u_{1}^{\prime}, x_{2} \mapsto u_{1}^{\prime \prime}, x_{3} \mapsto u_{2}$, onde $u_{2}$ é um monômio, temos que $u_{1}^{p-1}\left[u_{1}, u_{2}\right] u_{2}^{p-1} \in V$. 
Corolário 3.17. Sejam $f_{1}, f_{2}$ polinômios de $K\langle X\rangle$ tais que $f_{1}^{p-1}\left[f_{1}, f_{2}\right] f_{2}^{p-1}+V \neq 0$. Então,

$$
f_{1}^{p-1}\left[f_{1}, f_{2}\right] f_{2}^{p-1}+V=\sum_{i} \alpha_{i} x_{i_{1}}^{p-1}\left[x_{i_{1}}, x_{i_{2}}\right] x_{i_{2}}^{p-1}+f+V
$$

onde $x_{i} \in X$ para cada $i$ e $f$ pertence ao $T$-espaço de $K\langle X\rangle / V$ gerado por $\left[x_{1}, x_{2}\right]+V$.

Pelo corolário acima, todo elemento do $T$-subespaço gerado por

$$
q_{k}\left(x_{1}, \ldots, x_{2 k}\right)=x_{1}^{p-1}\left[x_{1}, x_{2}\right] x_{2}^{p-1} \cdots x_{2 k-1}^{p-1}\left[x_{2 k-1}, x_{2 k}\right] x_{2 k}^{p-1}
$$

será uma combinação linear de elementos do tipo

$$
x_{i_{1}}^{p-1}\left[x_{i_{1}}, x_{i_{2}}\right] x_{i_{2}}^{p-1} \cdots x_{i_{2 k-1}}^{p-1}\left[x_{i_{2 k-1}}, x_{i_{2 k}}\right] x_{i_{2 k}}^{p-1}+V
$$

e um polinômio $f$, onde $f$ pertence ao $T$-espaço de $K\langle X\rangle / V$ gerado por $\left[x_{1}, x_{2}\right]+V$.

Assim, temos o seguinte teorema (Ver [1] e [6]).

Teorema 3.18. O T-subespaço $C(H)$ dos polinômios centrais da álgebra de Grassmann não é finitamente gerado como T-espaço.

Demonstração. Seja $q_{k}\left(x_{1}, \ldots, x_{2 k}\right)=x_{1}^{p-1}\left[x_{1}, x_{2}\right] x_{2}^{p-1} \cdots x_{2 k-1}^{p-1}\left[x_{2 k-1}, x_{2 k}\right] x_{2 k}^{p-1}$. Pelo que foi dito acima, $q_{l}+V$ não pertence ao $T$-subespaço de $K\langle X\rangle / V$ gerado por $q_{1}+V, q_{2}+$ $V, \ldots, q_{l-1}+V, q_{l+1}+V, q_{l+2}+V, \ldots$ Logo, $C\left(G^{*}\right) / V$ não é finitamente gerado como $T$ subespaço. 


\section{Capítulo 4}

\section{Álgebra de Grassmann não-unitária de dimensão finita}

Trabalharemos nesse capítulo com a álgebra de Grassmann não unitária $H_{n}$, de um $K$-módulo livre de dimensão $n$, sobre um domínio de integridade $K$ de característica $p>2$.

\subsection{Identidades $\mathbb{Z}_{2}$-graduadas de $H_{n}$}

Primeiramente, observamos que como $H_{n} \subset H$, temos que $H$ satisfaz as identidades

$$
y_{1} y_{2}-y_{2} y_{1}, \quad y_{1} z_{1}-z_{1} y_{1}, \quad z_{1} z_{2}+z_{2} z_{1}, \quad y_{1}^{p}
$$

Para encontrar as identidades de $H_{n}$, definiremos o peso de um monômio de $K\langle Y, Z\rangle$.

Definição 4.1. Dizemos que os elementos de $Y$ em $K\langle Y, Z\rangle$ tem peso igual a 2 e os elementos de $Z$ tem peso igual a 1 . Se u é um monômio em $K\langle Y, Z\rangle$, o peso de u é a soma dos pesos das variáveis que aparecem em $u$. Denotaremos o peso de u por $\rho(u)$.

Exemplo 4.2. $\rho\left(y_{1} y_{2} z_{3}\right)=5, \rho\left(y_{1} z_{1} z_{2}\right)=4$.

Seja $f \in K\langle Y, Z\rangle$. Pelo corolário 3.1, $f+T_{2}(H)$ pode ser escrito unicamente como combinação linear dos elementos

$$
y_{i_{1}}^{m_{1}} \cdots y_{i_{k}}^{m_{k}} z_{j_{1}} \cdots z_{j_{l}}+T_{2}(H)
$$


onde $i_{1}<\ldots<i_{k}, j_{1}<\ldots<j_{l} ; 0<m_{1}, \ldots, m_{k}<p ; k, l \geq 0$ e $k+l>0$. Definimos $\rho_{\min }\left(f+T_{2}(H)\right)$ como o menor peso desses monômios $y_{i_{1}}^{m_{1}} \cdots y_{i_{k}}^{m_{k}} z_{j_{1}} \cdots z_{j_{l}} \in K\langle Y, Z\rangle$ que aparecem em $f+T_{2}(H)$.

Exemplo 4.3. $\rho_{\min }\left(y_{1} y_{2}+y_{1} z_{1}+T_{2}(H)\right)=3$, pois $\rho\left(y_{1} y_{2}\right)=4$ e $\rho\left(y_{1} z_{1}\right)=3$.

Agora, demonstraremos que monômios com peso suficientemente grande são identidades de $H_{n}$.

Proposição 4.4. Seja $u=y_{i_{1}}^{m_{1}} \ldots y_{i_{k}}^{m_{k}} z_{j_{1}} \ldots z_{j_{l}} \in K\langle Y, Z\rangle, i_{1}<\cdots<i_{k}, j_{1}<\cdots<j_{l}$, $0<m_{1}, \ldots, m_{k}<p, k+l>0, k, l \geq 0$. O monômio u é uma identidade $\mathbb{Z}_{2}$-graduada de $H_{n}$ se, e somente se, $\rho(u)>n$.

Demonstração. De fato, se $\rho(u) \leq n$, faça a seguinte substituição:

$$
\begin{aligned}
y_{i_{1}} & \mapsto e_{1} e_{2}+\cdots+e_{2 m_{1}-1} e_{2 m_{1}} \\
y_{i_{2}} & \mapsto e_{2 m_{1}+1} e_{2 m_{1}+2}+\cdots+e_{2\left(m_{1}+m_{2}\right)-1} e_{2\left(m_{1}+m_{2}\right)} \\
& \vdots \\
y_{i_{k}} & \mapsto e_{2\left(m_{1}+\cdots+m_{k-1}\right)+1} e_{2\left(m_{1}+\cdots+m_{k-1}\right)+2}+\cdots+e_{2\left(m_{1}+\cdots+m_{k}\right)-1} e_{2\left(m_{1}+\cdots+m_{k}\right)} \\
z_{j_{1}} & \mapsto e_{2\left(m_{1}+\cdots+m_{k}\right)+1} \\
& \vdots \\
z_{j_{l}} & \mapsto e_{2\left(m_{1}+\cdots+m_{k}\right)+l} .
\end{aligned}
$$

Logo $u \mapsto m_{1} ! \cdots m_{k} ! e_{1} \cdots e_{2\left(m_{1}+\cdots+m_{k}\right)+l}$. Como

$$
\rho(u)=2\left(m_{1}+\cdots+m_{k}\right)+l \leq n,
$$

temos que $u$ não é identidade de $H_{n}$.

Por outro lado, suponha que $\rho(u)>n$. Toda substituição dos $y_{i}$ e $z_{j}$ de $u$, por elementos pares e ímpares de $H_{n}$, respectivamente, será combinação linear de elementos da base de $H_{n}$ de comprimento maior que $n$. Como $H_{n}$ é gerada como álgebra por $e_{1}, \ldots, e_{n}$ e $e_{i}^{2}=0$ para $i=1, \ldots, n$, todo monômio de comprimento maior que $n$ é igual a 0 . Logo, $m$ é uma identidade se $\rho(u)>n$.

Agora, demonstraremos o seguinte 
Teorema 4.5. $K\langle Y, Z\rangle / T_{2}\left(H_{n}\right)$ é um $K$-módulo livre com uma $K$-base formada pelos monômios,

$$
y_{i_{1}}^{m_{1}} \ldots y_{i_{k}}^{m_{k}} z_{j_{1}} \ldots z_{j_{l}}+T_{2}\left(H_{n}\right)
$$

onde $2\left(m_{1}+\cdots+m_{k}\right)+l \leq n, k+l>0, k, l \geq 0 i_{1}<\cdots<i_{k}, j_{1}<\cdots<j_{l}$, $0<m_{1}, \ldots, m_{k}<p$.

Demonstração. Primeiro, $K$ possui um subcorpo de $p$ elementos. Portanto, como o grau de cada variável dos monômios do tipo (4.2) é menor que $p$, pela proposição 2.18 do capítulo 2, se uma combinação linear desses elementos está em $T_{2}(H)$, então cada elemento da forma (4.2) pertence a $T_{2}(H)$. Mas isso não pode acontecer, pela proposição anterior. Além disso, é fácil ver que esses elementos geram $K\langle Y, Z\rangle / T_{2}\left(H_{n}\right)$. Logo, os elementos do tipo (4.2) formam uma $K$-base de $K\langle Y, Z\rangle / T_{2}\left(H_{n}\right)$.

Teorema 4.6. As identidades $\mathbb{Z}_{2}$-graduadas de $H_{n}$ é o $T_{2}$-ideal de $K\langle Y, Z\rangle$ gerado por (4.1) e pelos monômios $u \operatorname{com} \rho(u)>n$.

Demonstração. De fato, se $f \in T_{2}\left(H_{n}\right)$ e $f \notin T_{2}(H), f$ é uma combinação linear de monômios (4.2) com peso maior que $n$.

Agora, seja $\mathcal{D}_{n}$ a subálgebra de $K\langle Y, Z\rangle / T_{2}\left(H_{n}\right)$ gerada por $x_{i}+T_{2}\left(H_{n}\right)$, onde $x_{i}=$ $y_{i}+z_{i}$. Como $T_{2}(H) \subset T_{2}\left(H_{n}\right)$, temos que $\mathcal{D}_{n}$ também satisfaz as identidades $\left[x_{1}, x_{2}, x_{2}\right]$ e $x_{1}^{p}$.

Seja $v_{n}$ o polinômio definido por

$$
v_{n}=\left(\cdots\left(\left(x_{1} \circ x_{2}\right) \circ x_{3}\right) \circ \cdots \circ x_{n-1}\right) \circ x_{n},
$$

onde $x_{1} \circ x_{2}=x_{1} x_{2}+x_{2} x_{1}$.

Proposição 4.7. $\rho_{\min }\left(v_{n+1}+T_{2}(H)\right)>2$ n para todo $n=1,2, \ldots$.

Demonstração. Vamos demonstrar por indução em $n$. Primeiro, para $n=1$ temos, mó- 
dulo $T_{2}(H)$

$$
\begin{aligned}
v_{2} & =x_{1} x_{2}+x_{2} x_{1} \\
& =\left(y_{1}+z_{1}\right)\left(y_{2}+z_{2}\right)+\left(y_{2}+z_{2}\right)\left(y_{1}+z_{1}\right) \\
& =2\left(y_{1} y_{2}+y_{1} z_{2}+y_{2} z_{1}\right)+z_{1} z_{2}+z_{2} z_{1} \\
& =2\left(y_{1} y_{2}+y_{1} z_{2}+y_{2} z_{1}\right) .
\end{aligned}
$$

Logo, $\rho_{\min }\left(v_{2}+T_{2}(H)\right)=3$. Agora suponha que $\rho_{\min }\left(v_{n}+T_{2}(H)\right)>2 n-2$. Escreva $v_{n}=v_{n}^{(0)}+v_{n}^{(1)}$, onde $v_{n}^{(0)}$ é a parte par de $v_{n}$ e $v_{n}^{(1)}$ é a parte ímpar de $v_{n}$. Assim temos que, módulo $T_{2}(H)$,

$$
\begin{aligned}
v_{n+1} & =v_{n} \circ x_{n+1} \\
& =v_{n} x_{n+1}+x_{n+1} v_{n} \\
& =v_{n}\left(y_{n+1}+z_{n+1}\right)+\left(y_{n+1}+z_{n+1}\right) v_{n} \\
& =2\left(v_{n} y_{n+1}\right)+v_{n} z_{n+1}+z_{n+1} v_{n} \\
& =2\left(v_{n} y_{n+1}\right)+\left(v_{n}^{(0)}+v_{n}^{(1)}\right) z_{n+1}+z_{n+1}\left(v_{n}^{(0)}+v_{n}^{(1)}\right) \\
& =2\left(v_{n} y_{n+1}\right)+2\left(v_{n}^{(0)} z_{n+1}\right) .
\end{aligned}
$$

Mas $\rho_{\min }\left(v_{n} y_{n+1}+T_{2}(H)\right)>(2 n-2)+2=2 n$. Também,

$$
\rho_{\min }\left(v_{n}^{(0)}+T_{2}(H)\right)>2 n-2
$$

e $\rho_{\min }\left(v_{n}^{(0)}+T_{2}(H)\right)$ é par, desde que monômios pares têm peso par. Portanto, $\rho_{\min }\left(v_{n}^{(0)}+\right.$ $\left.T_{2}(H)\right) \geq 2 n$. Assim, $\rho_{\min }\left(v_{n}^{(0)} z_{n+1}+T_{2}(H)\right)>2 n$.

Assim, temos os seguintes corolários.

Corolário 4.8. $\mathcal{D}_{2 n}$ satisfaz a identidade $v_{n+1}$.

Corolário 4.9. $\mathcal{D}_{2 n-1}$ satisfaz as identidades $v_{n} x_{n+1}$ e $x_{n+1} v_{n}$.

Demonstração. De fato, $\rho_{\min }\left(v_{n}+T_{2}(H)\right)>2 n-2$. Logo

$$
\rho_{\min }\left(v_{n} x_{n+1}+T_{2}(H)\right)=\rho_{\min }\left(x_{n+1} v_{n}+T_{2}(H)\right)>(2 n-2)+1=2 n-1 .
$$


Proposição 4.10. Se $s=n /(2 p-1)$ é um número inteiro, então $\mathcal{D}_{2 n-1}$ satisfaz a identidade

$$
\left[x_{1}, x_{2}\right] \cdots\left[x_{2 s-1}, x_{2 s}\right] x_{1}^{p-1} \cdots x_{2 s}^{p-1}
$$

Demonstração. De fato,

$$
s=n /(2 p-1) \Rightarrow 2(s(2 p-1))=2 n
$$

e

$$
\rho\left(\left[x_{1}, x_{2}\right] \cdots\left[x_{2 s-1}, x_{2 s}\right] x_{1}^{p-1} \cdots x_{2 s}^{p-1}+T_{2}(H)\right)=2(s(2 p-1)) .
$$

De fato,

$$
\begin{aligned}
{\left[x_{1}, x_{2}\right] x_{1}^{p-1} x_{2}^{p-1}+T_{2}(H) } & =2 z_{1} z_{2}\left(y_{1}^{p-1}+(p-1) y_{1}^{p-2} z_{1}\right)\left(y_{2}^{p-1}+(p-1) y_{2}^{p-2} z_{2}\right)+T_{2}(H) \\
& =2 y_{1}^{p-1} y_{2}^{p-1} z_{1} z_{2}+T_{2}(H)
\end{aligned}
$$

$\log 0$

$$
\left[x_{1}, x_{2}\right] \cdots\left[x_{2 s-1}, x_{2 s}\right] x_{1}^{p-1} \cdots x_{2 s}^{p-1}+T_{2}(H)=2^{s} y_{1}^{p-1} \cdots y_{2 s}^{p-1} z_{1} \cdots z_{2 s}+T_{2}(H)
$$

\subsection{Identidades de $H_{2 n}$}

Seja $V_{n+1}$ o $T$-ideal gerado por $V=T(H)$ e pelo polinômio $v_{n+1}$ em $K\langle X\rangle$. Vamos começar demonstrando alguns lemas.

Lema 4.11. $\left[x_{1} \cdots x_{n}, f\right]=\sum_{i=1}^{n}\left[x_{i}, f\right] x_{1} x_{2} \cdots \widehat{x}_{i} \cdots x_{n}$ módulo $T(H)$, onde $\widehat{x_{i}}$ significa que $x_{i}$ não aparece no produto.

Demonstração. Vamos demonstrar usando indução sobre $n$. Primeiro, vamos fazer para $n=2$.

$$
\begin{aligned}
{\left[x_{1} x_{2}, f\right]+T(H) } & =\left[x_{1}, f\right] x_{2}+x_{1}\left[x_{2}, f\right]+T(H) \\
& =\left[x_{1}, f\right] x_{2}+\left[x_{2}, f\right] x_{1}+T(H) .
\end{aligned}
$$


Aqui usamos o fato de que o comutador é central módulo $T(H)$.

Agora suponha que

$$
\left[x_{1} \cdots x_{n-1}, f\right]=\sum_{i=1}^{n-1}\left[x_{i}, f\right] x_{1} x_{2} \cdots \widehat{x}_{i} \cdots x_{n-1}
$$

Então

$$
\begin{aligned}
{\left[x_{1} \cdots x_{n}, f\right] } & =\left[x_{n}, f\right] x_{1} \cdots x_{n-1}+\left[x_{1} \cdots x_{n-1}, f\right] x_{n} \\
& =\left[x_{n}, f\right] x_{1} \cdots x_{n-1}+\left(\sum_{i=1}^{n-1}\left[x_{i}, f\right] x_{1} x_{2} \cdots \widehat{x}_{i} \cdots x_{n-1}\right) x_{n} \\
& =\left[x_{n}, f\right] x_{1} \cdots x_{n-1}+\sum_{i=1}^{n-1}\left[x_{i}, f\right] x_{1} x_{2} \cdots \widehat{x}_{i} \cdots x_{n-1} x_{n} \\
& =\sum_{i=1}^{n}\left[x_{i}, f\right] x_{1} x_{2} \cdots \widehat{x_{i}} \cdots x_{n} .
\end{aligned}
$$

Como $\left[x_{i}, f\right] x_{1} x_{2} \cdots \widehat{x}_{i} \cdots x_{n}$ tem $n$ fatores, $\left[x_{1} \cdots x_{n}, f\right]$ é um somatório de polinômios de $n$ fatores.

Corolário 4.12. $\left(x_{1} \cdots x_{n}\right) \circ x_{n+1}=2 x_{1} \cdots x_{n+1}-\sum_{i=1}^{n}\left[x_{i}, x_{n+1}\right] x_{1} x_{2} \cdots \widehat{x}_{i} \cdots x_{n}$ módulo $T(H)$.

Demonstração. De fato, $\left(x_{1} \cdots x_{n}\right) \circ x_{n+1}=2 x_{1} \cdots x_{n+1}-\left[x_{1} \cdots x_{n}, x_{n+1}\right]$.

Proposição 4.13. Módulo $T(H)$, temos que

$x_{1} \circ \ldots \circ x_{n+1}=2^{\eta} x_{1} \cdots x_{n+1}+\sum_{\substack{i_{1}<\ldots<i_{2 k} \\ i_{2 k+1}<\ldots<i_{n+1} \\ 1 \leq k \leq(n+1) / 2}} \xi_{i_{1} \cdots i_{n+1}} 2^{\eta_{k}}\left[x_{i_{1}}, x_{i_{2}}\right] \cdots\left[x_{i_{2 k-1}}, x_{i_{2 k}}\right] x_{i_{2 k+1}} \cdots x_{n+1}$

onde $\xi_{i_{1} \cdots i_{n+1}}= \pm 1, \eta, \eta_{k} \in \mathbb{N}$.

Demonstração. A demonstração será por indução sobre $n$. Para $n=2$,

$$
x_{1} \circ x_{2}=2 x_{1} x_{2}-\left[x_{1}, x_{2}\right]
$$


Suponhamos que

$$
x_{1} \circ \ldots \circ x_{n}=2^{\eta} x_{1} \cdots x_{n}+\sum_{\substack{i_{1}<\ldots<i_{2 k} \\ i_{2 k+1<\ldots<i i_{n}} \\ 1 \leq k \leq n / 2}} \xi_{i_{1} \cdots i_{n+1}} 2^{\eta_{k}}\left[x_{i_{1}}, x_{i_{2}}\right] \cdots\left[x_{i_{2 k-1}}, x_{i_{2 k}}\right] x_{i_{2 k+1}} \cdots x_{n} .
$$

Então,

$$
\begin{aligned}
& v_{n+1}=\left(x_{1} \circ \ldots \circ x_{n}\right) x_{n+1}+x_{n+1}\left(x_{1} \circ \ldots \circ x_{n}\right) \\
& =\left(2^{\eta} x_{1} \cdots x_{n}+\sum_{\substack{i_{1}<\ldots<i_{2 k} \\
i_{2 k+1}<\ldots<i_{n} \\
1 \leq k \leq n / 2}} \xi_{i_{1} \cdots i_{n+1}} 2^{\eta_{k}}\left[x_{i_{1}}, x_{i_{2}}\right] \cdots\left[x_{i_{2 k-1}}, x_{i_{2 k}}\right] x_{i_{2 k+1}} \cdots x_{n}\right) x_{n+1} \\
& +x_{n+1}\left(2^{\eta} x_{1} \cdots x_{n}+\sum_{\substack{i_{1}<\ldots<i_{2 k} \\
i_{2 k+1<\ldots<i n}<\ldots<i n \\
1 \leq k \leq n / 2}} \xi_{i_{1} \cdots i_{n+1}} 2^{\eta_{k}}\left[x_{i_{1}}, x_{i_{2}}\right] \cdots\left[x_{i_{2 k-1}}, x_{i_{2 k}}\right] x_{i_{2 k+1}} \cdots x_{n}\right) \\
& =2^{\eta}\left(x_{1} \cdots x_{n}\right) \circ x_{n+1}+ \\
& +\sum_{\substack{i_{1}<\ldots<i_{2 k} \\
i_{2 k+1}<\ldots<i_{n} \\
1 \leq k \leq n<2}} \xi_{i_{1} \cdots i_{n+1}} 2^{\eta_{k}}\left[x_{i_{1}}, x_{i_{2}}\right] \cdots\left[x_{i_{2 k-1}}, x_{i_{2 k}}\right]\left(\left(x_{i_{2 k+1}} \cdots x_{n}\right) \circ x_{n+1}\right) \\
& =2^{\eta+1} x_{1} \cdots x_{n+1}-2^{\eta} \sum_{j=1}^{n}\left[x_{j}, x_{n+1}\right] x_{1} x_{2} \cdots \widehat{x_{j}} \cdots x_{n}+
\end{aligned}
$$

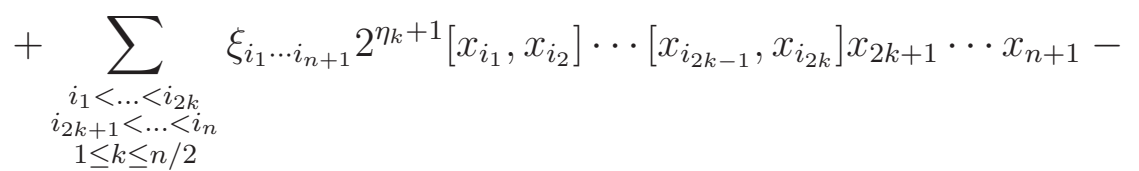

$$
\begin{aligned}
& -\sum_{\substack{i_{1}<\ldots<i_{2 k} \\
i_{2 k+1<\ldots<i n}<\ldots<i_{n} \\
1 \leq k \leq n / 2}}\left(\xi_{i_{1} \cdots i_{n+1}} 2^{\eta_{k}}\left[x_{i_{1}}, x_{i_{2}}\right] \cdots\left[x_{i_{2 k-1}}, x_{i_{2 k}}\right] \sum_{j=2 k+1}^{n}\left[x_{j}, x_{n+1}\right] x_{2 k+1} x_{2} \cdots \widehat{x}_{j} \cdots x_{n}\right) \\
& =2^{\eta+1} x_{1} \cdots x_{n+1}+\sum_{\substack{i_{1}<\ldots<i_{2 k^{\prime}} \\
i_{2 k^{\prime}+1<\ldots<i_{n}}<\ldots<\\
1 \leq k^{\prime} \leq n / 2}} \xi_{i_{1} \cdots i_{n+1}} 2^{\eta_{k}^{\prime}}\left[x_{i_{1}}, x_{i_{2}}\right] \cdots\left[x_{i_{2 k^{\prime}-1}}, x_{i_{2 k}}\right] x_{i_{2 k^{\prime}+1}} \cdots x_{i_{n+1}} .
\end{aligned}
$$

Corolário 4.14. Todo produto de elementos de $K\langle X\rangle$ pode ser reescrito, módulo $V_{n+1}$, como combinação linear de produtos com no máximo $n$ fatores.

Seja

$$
\begin{aligned}
B_{n+1}= & \left\{x_{i_{1}}^{m 1} \cdots x_{i_{k}}^{m_{k}}\left[x_{j_{1}}, x_{j_{2}}\right] \cdots\left[x_{j_{2 l-1}}, x_{j_{2 l}}\right] \mid i_{1}<\ldots<i_{k}, j_{1}<\ldots<j_{2 l}\right. \\
& \left.0<m_{1}, \ldots, m_{k}<p, k+l>0, m_{1}+\ldots+m_{k}+l \leq n\right\}
\end{aligned}
$$


Corolário 4.15. O conjunto $\left\{b+V_{n+1} \mid b \in B_{n+1}\right\}$ gera $K\langle X\rangle / V_{n+1}$.

Demonstração. Como já vimos, módulo $V$, o conjunto de polinômios

$$
x_{i_{1}}^{m 1} \cdots x_{i_{k}}^{m_{k}}\left[x_{j_{1}}, x_{j_{2}}\right] \cdots\left[x_{j_{2 l-1}}, x_{j_{2 l}}\right]
$$

onde $i_{1}<\ldots<i_{k}, j_{1}<\ldots<j_{2 l}, 0<m_{1}, \ldots, m_{k}<p, k+l>0$, gera $K\langle X\rangle / V$. Como $V \subset V_{n+1}$, temos que (4.3) gera $K\langle X\rangle / V_{n+1}$. Pelo corolário 4.14, os elementos de 4.3 podem ser reescritos como combinação linear de produtos com no máximo $n$ fatores. Logo, $\left\{b+V_{n+1} \mid b \in B_{n+1}\right\}$ gera $K\langle X\rangle / V_{n+1}$.

Seja

$$
\begin{aligned}
\phi: K\langle X\rangle \longrightarrow \mathcal{D}_{2 n} \\
x_{i} \mapsto x_{i}+T_{2}\left(H_{2 n}\right)
\end{aligned}
$$

isto é, $\phi\left(f\left(x_{1}, \ldots, x_{n}\right)\right)=f\left(x_{1}, \ldots, x_{n}\right)+T_{2}\left(H_{2 n}\right)$. Pelo corolário 4.8, o $T$-ideal $V_{n+1}$ está contido no núcleo de $\Phi$. Logo,

$$
\begin{array}{r}
\Phi: K\langle X\rangle / V_{n+1} \longrightarrow \mathcal{D}_{2 n} \\
f\left(x_{1}, \ldots, x_{n}\right)+V_{n+1} \mapsto f\left(x_{1}, \ldots, x_{n}\right)+T_{2}\left(H_{2 n}\right)
\end{array}
$$

está bem definido.

Proposição 4.16. Os elementos de $\mathcal{D}_{2 n}$ da forma

$$
b+T_{2}\left(H_{2 n}\right)
$$

onde $x_{i}=y_{i}+z_{i}$, são linearmente independentes em $\mathcal{D}_{2 n}$.

Demonstração. A prova é análoga à proposição 3.5, pois

$x_{i_{1}}^{m_{1}} \cdots x_{i_{k}}^{m_{k}}\left[x_{j_{1}}, x_{j_{2}}\right] \cdots\left[x_{j_{2 l-1}}, x_{j_{2 l}}\right]+T_{2}\left(H_{2 n}\right)=2^{l} y_{i_{1}}^{m_{1}} \cdots y_{i_{k}}^{m_{k}} z_{j_{1}} \cdots z_{j_{2 l}}+\cdots+T_{2}\left(H_{2 n}\right)$

e $\rho\left(y_{i_{1}}^{m_{1}} \cdots y_{i_{k}}^{m_{k}} z_{j_{1}} \cdots z_{j_{2 l}}\right)=2\left(m_{1}+\ldots+m_{k}+l\right) \leq 2 n$, isto é,

$$
y_{i_{1}}^{m_{1}} \cdots y_{i_{k}}^{m_{k}} z_{j_{1}} \cdots z_{j_{2 l}}+T_{2}\left(H_{2 n}\right) \neq T_{2}\left(H_{2 n}\right)
$$


Logo, temos o seguinte corolário.

Corolário 4.17. O homomorfismo

$$
\begin{array}{r}
\Phi: K\langle X\rangle / V_{n+1} \rightarrow \mathcal{D}_{2 n} \\
f\left(x_{1}, \ldots, x_{n}\right)+V_{n+1} \mapsto f\left(x_{1}, \ldots, x_{n}\right)+T_{2}\left(H_{2 n}\right)
\end{array}
$$

é um isomorfismo.

Logo $K\langle X\rangle / V_{n+1} \simeq \mathcal{D}_{2 n} \simeq K\langle X\rangle / T\left(H_{2 n}\right)$. Portanto, temos o seguinte teorema.

Teorema 4.18. O T-ideal da identidades de $H_{2 n}$ é o T-ideal gerado pelos polinômios $\left[x_{1}, x_{2}, x_{3}\right], x_{1}^{p}$ e $v_{n+1}$.

\subsection{Identidades de $H_{2 n-1}$}

Definição 4.19. $W_{n+1}$ é o $T$-ideal de $K\langle X\rangle$ gerado por $T(H)$ e pelos polinômios

$$
x_{n+1} v_{n}, v_{n} x_{n+1}
$$

e se $s=n /(2 p-1)$ é um número inteiro incluímos o polinômio

$$
\left[x_{1}, x_{2}\right] \cdots\left[x_{2 s-1}, x_{2 s}\right] x_{1}^{p-1} \cdots x_{2 s}^{p-1} .
$$

Considere o seguinte homomorfismo

$$
\begin{aligned}
\phi & : K\langle X\rangle \rightarrow \mathcal{D}_{2 n-1} \\
x_{i} & \mapsto x_{i}+T_{2}\left(H_{2 n-1}\right),
\end{aligned}
$$

isto é, $\phi\left(f\left(x_{1}, \ldots, x_{n}\right)\right)=f\left(x_{1}, \ldots, x_{n}\right)+T_{2}\left(H_{2 n-1}\right)$.

Pelo corolário 4.9 e proposição 4.10 , temos que

$$
\begin{array}{r}
\Phi: K\langle X\rangle / W_{n+1} \rightarrow \mathcal{D}_{2 n-1} \\
f\left(x_{1}, \ldots, x_{n}\right)+W_{n+1} \mapsto f\left(x_{1}, \ldots, x_{n}\right)+T_{2}\left(H_{2 n-1}\right)
\end{array}
$$

está bem definido, pois $W_{n+1} \subset \operatorname{Ker}(\phi)$. 
Vamos mostrar agora que $\Phi$ é um isomorfismo. Para isso, vamos demonstrar alguns fatos.

Primeiro, vamos encontrar um conjunto gerador para $K\langle X\rangle / W_{n+1}$. Como $x_{n+1} v_{n}$ e $v_{n} x_{n+1}$ pertencem a $W_{n+1}$, temos que

$$
v_{n+1}=x_{n+1} v_{n}+v_{n} x_{n+1} \in W_{n+1} .
$$

isto é, $V_{n+1} \subset W_{n+1}$. Logo temos o seguinte lema.

Lema 4.20. O conjunto $\left\{b+W_{n+1} \mid b \in B_{n+1}\right\}$ gera $K\langle X\rangle / W_{n+1}$.

Demonstração. O conjunto $\left\{b+V_{n+1} \mid b \in B_{n+1}\right\}$ gera $K\langle X\rangle / V_{n+1}$. Como $V_{n+1} \subset W_{n+1}$, temos que $\left\{b+W_{n+1} \mid b \in B_{n+1}\right\}$ gera $K\langle X\rangle / W_{n+1}$.

A partir de agora escreveremos o conjunto $B_{n+1}$ da seguinte forma

$$
\begin{aligned}
B_{n+1}= & \left\{x_{i_{1}}^{\epsilon_{1}} \cdots x_{i_{k}}^{\epsilon_{k}}\left[x_{j_{1}}, x_{j_{2}}\right] \cdots\left[x_{j_{2 l-1}}, x_{j_{2 l}}\right] x_{j_{1}}^{\delta_{1}} \cdots x_{j_{2 l}}^{\delta_{2 l}} \mid i_{1}<\ldots<i_{k}, j_{1}<\ldots<j_{2 l},\right. \\
& 0<\epsilon_{1}, \ldots, \epsilon_{k}<p, 0 \leq \delta_{1}, \ldots, \delta_{2 l}<p, i_{r} \neq j_{s}(1 \leq r \leq k, 1 \leq s \leq 2 l,) k+l>0, \\
& \left.\epsilon_{1}+\ldots+\epsilon_{k}+\delta_{1}+\ldots+\delta_{2 l}+l \leq n\right\} .
\end{aligned}
$$

Escreveremos $B_{n+1}$ dessa maneira, pois módulo $W_{n+1}$, toda variável que pertence a parte comutador comuta com qualquer outra variável. Por exemplo, módulo $W_{n+1}$,

$$
\begin{aligned}
x_{1} x_{2}\left[x_{1}, x_{3}\right] & =\left(x_{2} x_{1}+\left[x_{1}, x_{2}\right]\right)\left[x_{1}, x_{3}\right] \\
& =x_{2} x_{1}\left[x_{1}, x_{3}\right]+\left[x_{1}, x_{2}\right]\left[x_{1}, x_{3}\right] \\
& =x_{2} x_{1}\left[x_{1}, x_{3}\right] \\
& =x_{2}\left[x_{1}, x_{3}\right] x_{1},
\end{aligned}
$$

isto é, podemos colocar, módulo $W_{n+1}$, as variáveis que pertencem à parte comutador por último.

Como já foi dito, todo elemento de $B_{n+1}$ tem no máximo $n$ fatores. Considere então a seguinte definição, que pode ser encontrada em [34].

Definição 4.21. Seja $B_{n+1}^{\prime}$ o subconjunto de $B_{n+1}$ formado pelos elementos:

i) $b \in B_{n+1}$ com número de fatores menor que $n$, isto é,

$$
b=x_{i_{1}}^{\epsilon_{1}} \cdots x_{i_{k}}^{\epsilon_{k}}\left[x_{j_{1}}, x_{j_{2}}\right] \cdots\left[x_{j_{2 l-1}}, x_{j_{2 l}}\right] x_{j_{1}}^{\delta_{1}} \cdots x_{j_{2 l}}^{\delta_{2 l}}
$$


onde $i_{1}<\ldots<i_{k}, j_{1}<\ldots<j_{2 l}, 0<m_{1}, \ldots, m_{k}<p, k+l>0$ e $m_{1}+\ldots+m_{k}+l<$ $n$.

ii) $S e b=x_{i_{1}}^{\epsilon_{1}} \cdots x_{i_{k}}^{\epsilon_{k}}\left[x_{j_{1}}, x_{j_{2}}\right] \cdots\left[x_{j_{2 l-1}}, x_{j_{2 l}}\right] x_{j_{1}}^{\delta_{1}} \cdots x_{j_{2 l}}^{\delta_{2 l}} \in B_{n+1}$ tem $n$ fatores, então $x_{i_{1}}$ é a variável de menor índice que tem grau menor que $p$, isto é, toda variável $x_{j_{t}}$ com $j_{t}<i_{1}$ tem grau $p$.

O seguinte lema pode ser encontrado em [35].

Lema 4.22. Seja $b \in B_{n+1}$ um elemento com $n$ fatores. Então $b$ pode ser reescrito, módulo $W_{n+1}$, como combinação linear de elementos de $B_{n+1}^{\prime}$.

Pelos dois últimos lemas temos

Proposição 4.23. O conjunto $\left\{b+W_{n+1} \mid b \in B_{n+1}^{\prime}\right\}$ gera $K\langle X\rangle / W_{n+1}$.

Seja $b=x_{i_{1}}^{\epsilon_{1}} \cdots x_{i_{k}}^{\epsilon_{k}}\left[x_{j_{1}}, x_{j_{2}}\right] \cdots\left[x_{j_{2 l-1}}, x_{j_{2 l}}\right] x_{j_{1}}^{\delta_{1}} \cdots x_{j_{2 l}}^{\delta_{2 l}} \in B_{n+1}^{\prime}$, onde $b$ tem $n$ fatores, isto é, $\left(\epsilon_{1}+\ldots \epsilon_{k}+\delta_{1}+\ldots+\delta_{2 l}+l\right)=n$. Assim, em $\mathcal{D}_{2 n-1}$,

$b+T_{2}\left(H_{2 n-1}\right)=2^{l}\left(y_{i_{1}}^{\epsilon_{1}}+\epsilon_{1} y_{i_{1}}^{\epsilon_{1}-1} z_{i_{1}}\right) \cdots\left(y_{i_{k}}^{\epsilon_{k}}+\epsilon_{k} y_{i_{k}}^{\epsilon_{k}-1} z_{i_{k}}\right) z_{j_{1}} \cdots z_{j_{2 l}} y_{j_{1}}^{\delta_{1}} \cdots y_{j_{2 l}}^{\delta_{2 l}}+T_{2}\left(H_{2 n-1}\right)$.

Note que o monômio de maior peso que aparece em $b+T_{2}\left(H_{2 n-1}\right)$ é

$$
y_{i_{1}}^{\epsilon_{1}} \cdots y_{i_{k}}^{\epsilon_{k}} y_{j_{1}}^{\delta_{1}} \cdots y_{j_{2 l}}^{\delta_{2 l}} z_{j_{1}} \cdots z_{2 l}+T_{2}\left(H_{2 n-1}\right)
$$

Como $\rho\left(y_{i_{1}}^{\epsilon_{1}} \cdots y_{i_{k}}^{\epsilon_{k}} y_{j_{1}}^{\delta_{1}} \cdots y_{j_{2 l}}^{\delta_{2 l}} z_{j_{1}} \cdots z_{2 l}\right)=2\left(\epsilon_{1}+\ldots+\epsilon_{k}+\delta_{1}+\ldots+\delta_{2 l}+l\right)=2 n$, temos que

$$
y_{i_{1}}^{\epsilon_{1}} \cdots y_{i_{k}}^{\epsilon_{k}} y_{j_{1}}^{\delta_{1}} \cdots y_{j_{2 l}}^{\delta_{2 l}} z_{j_{1}} \cdots z_{2 l}+T_{2}\left(H_{2 n-1}\right)=0 .
$$

A lista de monômios com peso igual a $2 n-1$ é

$$
\begin{gathered}
2^{l} \epsilon_{1} y_{i_{1}}^{\epsilon_{1}-1} y_{i_{2}}^{\epsilon_{2}} y_{i_{3}}^{\epsilon_{3}} \cdots y_{i_{k}}^{\epsilon_{k}} y_{j_{1}}^{\delta_{1}} \cdots y_{j_{2 l}}^{\delta_{2 l}} z_{i_{1}} z_{j_{1}} \cdots z_{2 l}, \\
2^{l} \epsilon_{2} y_{i_{1}}^{\epsilon_{1}} y_{i_{2}}^{\epsilon_{2}-1} y_{i_{3}}^{\epsilon_{3}} \cdots y_{i_{k}}^{\epsilon_{k}} y_{j_{1}}^{\delta_{1}} \cdots y_{j_{2 l}}^{\delta_{2 l}} z_{i_{2}} z_{j_{1}} \cdots z_{2 l}, \\
2^{l} \epsilon_{3} y_{i_{1}}^{\epsilon_{1}} y_{i_{2}}^{\epsilon_{2}} y_{i_{3}}^{\epsilon_{3}-1} \cdots y_{i_{k}}^{\epsilon_{k}} y_{j_{1}}^{\delta_{1}} \cdots y_{j_{2 l} l}^{\delta_{2 l}} z_{i_{3}} z_{j_{1}} \cdots z_{2 l}, \\
\vdots \\
2^{l} \epsilon_{k} y_{i_{1}}^{\epsilon_{1}} y_{i_{2}}^{\epsilon_{2}} y_{i_{3}}^{\epsilon_{3}} \cdots y_{i_{k}}^{\epsilon_{k}-1} y_{j_{1}}^{\delta_{1}} \cdots y_{j_{2 l}}^{\delta_{2 l}} z_{i_{k}} z_{j_{1}} \cdots z_{2 l} .
\end{gathered}
$$


Nessa lista, escolhemos o monômio líder como sendo $y_{i_{1}}^{\epsilon_{1}-1} y_{i_{2}}^{\epsilon_{2}} y_{i_{3}}^{\epsilon_{3}} \cdots y_{i_{k}}^{\epsilon_{k}} y_{j_{1}}^{\delta_{1}} \cdots y_{j_{2 l}}^{\delta_{2 l}} z_{i_{1}} z_{j_{1}} \cdots z_{2 l}$. Vamos mostrar que nenhum outro polinômio de $\left\{b+T_{2}\left(H_{2 n-1}\right) \mid b \in B_{n+1}^{\prime}\right\}$ tem

$$
y_{i_{1}}^{\epsilon_{1}-1} y_{i_{2}}^{\epsilon_{2}} y_{i_{3}}^{\epsilon_{3}} \cdots y_{i_{k}}^{\epsilon_{k}} y_{j_{1}}^{\delta_{1}} \cdots y_{j_{2 l}}^{\delta_{2 l}} z_{i_{1}} z_{j_{1}} \cdots z_{2 l}
$$

como monômio líder.

Primeiro, um polinômio com menos de $n$ fatores não pode ter um monômio com peso igual a $2 n-1$. De fato, se um polinômio $b+T_{2}\left(H_{2 n-1}\right)$, onde $b \in B_{n+1}^{\prime}$, tem $n-1$ fatores, então o monômio de maior peso que aparece em $b+T_{2}\left(H_{2 n-1}\right)$ tem peso igual a $2 n-2$.

Agora note que se (4.5) é o monômio líder de um outro polinômio $b^{\prime}+T_{2}\left(H_{2 n-1}\right)$, onde $b^{\prime}$ tem $n$ fatores, então no conjunto $\left\{x_{i_{1}}, x_{j_{1}}, \ldots, x_{j_{2 l}}\right\}, 2 l$ variáveis pertencem à parte comutador e uma variável não pertence à parte comutador de $b^{\prime}$. De fato, o número de $z^{\prime} s$ em (4.5) é $2 l+1$, no qual podemos formar no máximo $l$ comutadores usando a relação $\left[x_{i}, x_{j}\right]=2 z_{i} z_{j}$. Suponha que formamos $l-1$ comutadores, digamos $\left[x_{j_{3}}, x_{j_{4}}\right] \cdots\left[x_{j_{2 l-1}}, x_{j_{2 l}}\right]$. Logo, a menos de múltiplo, (4.5) é

$$
y_{i_{1}}^{\epsilon_{1}-1} y_{i_{2}}^{\epsilon_{2}} y_{i_{3}}^{\epsilon_{3}} \cdots y_{i_{k}}^{\epsilon_{k}} y_{j_{1}}^{\delta_{1}} \cdots y_{j_{2 l}}^{\delta_{2 l}} z_{i_{1}} z_{j_{1}} z_{j_{2}}\left[x_{j_{3}}, x_{j_{4}}\right] \cdots\left[x_{j_{2 l-1}}, x_{j_{2 l}}\right]
$$

Agora, com o índice $i_{1}$ sobrou $y_{i_{1}}^{\epsilon_{1}-1} z_{i_{1}}$. Logo $x_{i_{1}}^{\epsilon_{1}}$ tem que aparecer em $b^{\prime}+T_{2}\left(H_{2 n-1}\right)$. Analogamente, $y_{j_{1}}^{\delta_{1}} z_{j_{1}}$ e $y_{j_{2}}^{\delta_{2}} z_{j_{2}}$ implica que $x_{j_{1}}^{\delta_{1}+1}$ e $x_{j_{2}}^{\delta_{1}+1}$ tem que aparecer em $b^{\prime}+T_{2}\left(H_{2 n-1}\right)$, respectivamente. Assim, os seguintes fatores compõe $b^{\prime}+T_{2}\left(H_{2 n-1}\right)$ :

$$
x_{i_{1}}^{\epsilon_{1}}, x_{i_{2}}^{\epsilon_{2}}, \ldots, x_{i_{k}}^{\epsilon_{k}}, x_{j_{1}}^{\delta_{1}+1}, x_{j_{2}}^{\delta_{1}+1}, x_{j_{3}}^{\delta_{3}}, \cdots, x_{j_{2 l}}^{\delta_{2 l}},\left[x_{j_{3}}, x_{j_{4}}\right], \cdots,\left[x_{j_{2 l-1}}, x_{j_{2 l}}\right] .
$$

Mas isso implica que $b^{\prime}$ tem $\epsilon_{1}+\ldots+\epsilon_{k}+\left(\delta_{1}+1\right)+\left(\delta_{2}+1\right)+\delta_{3}+\ldots+\delta_{2 l}+(l-1)=n+1$ fatores.

Logo, (4.5) aparece em $b^{\prime}+T_{2}\left(H_{2 n-1}\right)$ se no conjunto $\left\{x_{i_{1}}, x_{j_{1}}, \ldots, x_{j_{2 l}}\right\}, 2 l$ variáveis pertencem à parte comutador e uma variável não pertence à parte comutador de $b^{\prime}$, pois $b^{\prime}$ tem $n$ fatores.

Seja $x_{t} \in\left\{x_{i_{1}}, x_{j_{1}}, \ldots, x_{j_{2 l}}\right\}$ a variável que não aparece na parte comutador de $b^{\prime}+$ $T_{2}\left(H_{2 n-1}\right)$. Se $t=i_{1}$, então $b^{\prime}=b$. Caso contrário, temos dois casos:

i) $t<i_{1}$. Nesse caso $x_{t}$ tem grau $p$. Como $x_{t}$ aparece na parte não comutador de $b^{\prime}+T_{2}\left(H_{2 n-1}\right)$ e $x_{t}^{p}+T_{2}\left(H_{2 n-1}\right)=0$, temos que $b^{\prime}+T_{2}\left(H_{2 n-1}\right)=0$. 
ii) $i_{1}<t$. Nesse caso, $x_{i_{1}}$ aparece na parte comutador de $b^{\prime}$ e $x_{t}$ na parte não-comutador de $b^{\prime}$. Logo $x_{i_{1}}$ teria que ter grau $p$, o que não ocorre.

Com o que foi dito acima, temos o seguinte teorema.

Teorema 4.24. O conjunto $\left\{b+T_{2}\left(H_{2 n-1}\right) \mid b \in B_{n+1}^{\prime}\right\}$ forma uma $K$-base para a álgebra $\mathcal{D}_{2 n-1}$.

Demonstração. Basta considerarmos os elementos de $\left\{b+T_{2}\left(H_{2 n-1}\right) \mid b \in B_{n+1}^{\prime}\right\}$ com $n$ fatores. Seja então

$$
\sum \alpha_{i} b_{i}+T_{2}\left(H_{2 n-1}\right)=0
$$

onde $b_{i} \in B_{n+1}^{\prime}$ possuem $n$ fatores. Como cada $b_{i}$ tem um monômio líder de peso $2 n-1$ unicamente determinado por $b_{i}$, temos que todos os $\alpha_{i}^{\prime} s$ são iguais a zero.

Logo, temos o seguinte corolário

Corolário 4.25. O homomorfismo

$$
\begin{array}{r}
\Phi: K\langle X\rangle / W_{n+1} \longrightarrow \mathcal{D}_{2 n-1} \\
f\left(x_{1}, \ldots, x_{n}\right)+W_{n+1} \mapsto f\left(x_{1}, \ldots, x_{n}\right)+T_{2}\left(H_{2 n-1}\right)
\end{array}
$$

é um isomorfismo.

Logo temos $K\langle X\rangle / W_{n+1} \simeq \mathcal{D}_{2 n-1} \simeq K\langle X\rangle / W_{n+1}$. Portanto, temos

Teorema 4.26. O T-ideal das identidades de $H_{2 n-1}$ é igual a $W_{n+1}$. 


\section{Capítulo 5}

\section{Álgebra de Grassmann unitária de dimensão infinita}

Nesse capítulo, estudaremos a álgebra de Grassmann unitária $E$ de dimensão infinita sobre um corpo $K$ finito. Se $|K|=q$, sabemos que $q=p^{n}$, onde $p$ é a característica de $K$ e $n$ é um inteiro positivo. Consideraremos $p>2$. Por [3], sabemos que o $T$-ideal de $K_{1}\langle X\rangle$ gerado por $\left[x_{1}, x_{2}, x_{3}\right]$ e $x_{1}^{q p}-x_{1}^{p}$ é o $T$-ideal das identidades de $E$. Daremos uma nova representação para álgebra $K\langle X\rangle / T(H)$, como produto tensorial de uma álgebra comutativa $A$ e uma álgebra $B$. Isso nos permite dar uma demonstração simples do resultado de [3].

Sejam $U=\left\langle x^{q p}-x^{p},\left[x_{1}, x_{2}, x_{3}\right]\right\rangle^{T}$ em $K_{1}\langle X\rangle, A=K_{1}\left[t_{i} \mid i \in \Lambda\right] / I$, onde $I$ é o ideal gerado por $t_{i}^{q}-t_{i}$, e $B=K_{1}\langle Y\rangle / V$, onde $V=\left\langle y^{p},\left[y_{1}, y_{2}, y_{3}\right]\right\rangle^{T}$ em $K\langle Y\rangle$.

Teorema 5.1. $K_{1}\langle X\rangle / U \simeq A \bigotimes_{K} B$.

Antes de demonstrarmos o teorema (5.1), mencionaremos alguns resultados já conhecidos.

Sejam

$$
\begin{gathered}
\mathcal{B}=\left\{x_{i_{1}}^{m_{1}} \cdots x_{i_{k}}^{m_{k}}\left[x_{j_{1}}, x_{j_{2}}\right] \cdots\left[x_{j_{2 l-1}}, x_{j_{2 l}}\right] \mid i_{1}<\ldots<i_{k}, j_{1}<\ldots<j_{2 l},\right. \\
\left.0<m_{i}<p, k \geq 0, l \geq 0, k^{2}+l^{2}>0\right\}
\end{gathered}
$$




$$
\mathcal{B}^{\prime}=\left\{x_{i_{1}^{\prime}}^{p m_{1}^{\prime}} \cdots x_{i_{k^{\prime}}^{\prime}}^{p m_{k^{\prime}}^{\prime}} \cdot b \mid \quad b \in \mathcal{B}, 0<m_{i^{\prime}}^{\prime}<q, k^{\prime} \geq 0\right\}
$$

Seja $H$ a álgebra de Grassmann de dimensão infinita, não unitária, sobre $K$. Pelo capítulo 2, sabemos que o $T$-ideal das identidades de $H$ é $V$, e que uma base de $K\langle Y\rangle / V$ como $K$-espaço vetorial é $\mathcal{B}(\bmod V)$.

Outro resultado conhecido é que $\left[x_{1}^{p}, x_{2}\right] \in U$, isto é, $x_{1}^{p}+U$ é central em $K_{1}\langle X\rangle / U$. Logo, vemos que $\mathcal{B}^{\prime}(\bmod U)$ gera $K_{1}\langle X\rangle / U$.

Proposição 5.2. $I=\left\langle t_{1}^{q}-t_{1}\right\rangle^{T}$ em $K_{1}\left[t_{i} \mid i \in \Lambda\right]$.

Demonstração. Primeiro, a incusão $I \subseteq\left\langle t_{1}^{q}-t_{1}\right\rangle^{T}$ é obvia. Seja $f \in\left\langle t_{1}^{q}-t_{1}\right\rangle^{T}$. Então,

$$
f=\sum f_{i_{0}}\left(f_{i_{1}}^{q}-f_{i_{1}}\right) f_{i_{2}}
$$

onde $f_{i_{0}}, f_{i_{1}}, f_{i_{2}} \in K_{1}\left[t_{i} \mid i \in \Lambda\right]$. Desde que para dois monômios quaisquer $u$ e $v,(u+v)^{q}=$ $u^{q}+v^{q}$, temos que

$$
f=\sum f_{i_{0}}\left(u_{i_{1}}^{q}-u_{i_{1}}\right) f_{i_{2}}
$$

onde $u_{i_{1}}$ são monômios. Logo, para mostrar a outra inclusão, resta mostrar que para todo monômio $u, u^{q}-u \in I$. Faremos isso por indução no comprimento $|u|$ de $u$. Se $|u|=1$, então $u=t_{i}$, para algum $i$. Logo, $u^{q}-u \in I$. Suponha agora que $|u|=k$ e que $u^{q}-u \in I$. Então,

$\left(u^{q}-u\right)\left(t_{i}^{q}+t_{i}\right)=u^{q} t_{i}^{q}-u t_{i}+u^{q} t_{i}-u t_{i}^{q} \in I\left(u^{q}+u\right)\left(t_{i}^{q}-t_{i}\right)=u^{q} t_{i}^{q}-u t_{i}-u^{q} t_{i}+u t_{i}^{q} \in I$

Somando o segundo membro das duas equações, temos

$$
2\left(u^{q} t_{i}^{q}-u t_{i}\right) \in I
$$

e desde que a característica de $K$ é diferente de 2 ,

$$
\left(u t_{i}\right)^{q}-u t_{i} \in I,
$$

o que completa a demonstração. 
Agora, $A$ e $B$ pertencem a variedade determinada por

$$
x^{q p}-x^{p},\left[x_{1}, x_{2}, x_{3}\right]
$$

e desde que $A$ é comutativa, $A \bigotimes_{K} B$ também satisfaz

$$
x^{q p}-x^{p},\left[x_{1}, x_{2}, x_{3}\right] .
$$

Para ver isso, seja $f_{1}, f_{2}, f_{3} \in A$ e $g_{1}, g_{2}, g_{3} \in B$. Então,

$$
\left[f_{1} \otimes g_{1}, f_{2} \otimes g_{2}, f_{3} \otimes g_{3}\right]=f_{1} f_{2} f_{3} \otimes\left[g_{1}, g_{2}, g_{3}\right]=0
$$

e

$$
\begin{aligned}
\left(f_{1} \otimes g_{1}\right)^{q p}-\left(f_{1} \otimes g_{1}\right)^{p} & =f_{1}^{q p} \otimes g_{1}^{q p}-f_{1}^{p} \otimes g_{1}^{p} \\
& =f_{1}^{p} \otimes g_{1}^{q p}-f_{1}^{p} \otimes g_{1}^{p} \\
& =f_{1}^{q p} \otimes\left(g_{1}^{q p}-g_{1}^{p}\right) \\
& =0 .
\end{aligned}
$$

Demostração do Teorema 5.1:

Considere o seguinte homomorfismo

$$
\begin{gathered}
\Phi: K_{1}\langle X\rangle / U \longrightarrow A \otimes B \\
\Phi\left(x_{i}+U\right)=\left(t_{i}^{r}+I\right) \otimes(1+V)-(1+I) \otimes\left(y_{i}+V\right),
\end{gathered}
$$

onde $r=p^{n-1}$. Como $K_{1}\langle X\rangle / U$ é a álgebra relativamente livre da variedade determinada por

$$
x^{q p}-x^{p},\left[x_{1}, x_{2}, x_{3}\right],
$$

basta definir $\Phi$ nos geradores livres $x_{i}+U$. Mostraremos que $\Phi$ é isomorfismo.

Primeiro mostraremos que $\Phi$ é sobrejetora. Procederemos da seguinte maneira: primeiro provaremos que os elementos $\left(t_{i}^{m}+I\right) \otimes(1+V)$, com $m<q$ pertencem a imagem 
da $\Phi$. Isso implicará que $A \bigotimes\{1+V\}$ pertence a imagem de $\Phi$. A seguir, mostraremos que os elementos $(1+I) \otimes\left(y_{i}+V\right)$ e $(1+I) \otimes\left(\left[y_{i}, y_{j}\right]+V\right)$ pertencem a imagem de $\Phi$, o que implicará que $\{1+I\} \otimes B$ pertence a imagem de $\phi$.

$$
\begin{aligned}
\Phi\left(x_{i}^{p}+U\right) & =\Phi\left(x_{i}+U\right)^{p} \\
& =\left(\left(t_{i}^{r}+I\right) \otimes(1+V)-(1+I) \otimes\left(y_{i}+V\right)\right)^{p} \\
& =\left(\left(t_{i}^{r}+I\right) \otimes(1+V)\right)^{p}-\left((1+I) \otimes\left(y_{i}+V\right)\right)^{p} \\
& =\left(t_{i}^{r p}+I\right) \otimes(1+V)-(1+I) \otimes\left(y_{i}^{p}+V\right) \\
& =\left(t_{i}^{q}+I\right) \otimes(1+V) \\
& =\left(t_{i}+I\right) \otimes(1+V) .
\end{aligned}
$$

Assim para $0<m<q$,

$$
\Phi\left(x_{i}^{p m}+U\right)=\left(t_{i}^{m}+I\right) \otimes(1+V)
$$

Além disso,

$$
\begin{aligned}
\Phi\left(x_{i}^{q}-x_{i}+U\right) & =\Phi\left(x_{i}^{q}+U\right)-\Phi\left(x_{i}+U\right) \\
& =\Phi\left(x_{i}^{p r}+U\right)-\Phi\left(x_{i}+U\right) \\
& =\Phi\left(x_{i}^{p}+U\right)^{r}-\Phi\left(x_{i}+U\right) \\
& =\left(t_{i}^{r}+I\right) \otimes(1+V)-\left(t_{i}^{r}+I\right) \otimes(1+V)+(1+I) \otimes\left(y_{i}+V\right) \\
& =(1+I) \otimes\left(y_{i}+V\right)
\end{aligned}
$$

e

$$
\begin{aligned}
\Phi\left(\left[x_{i}, x_{j}\right]+U\right)= & \Phi\left(x_{i}+U\right) \Phi\left(x_{j}+U\right)-\Phi\left(x_{j}+U\right) \Phi\left(x_{i}+U\right) \\
= & \left(\left(t_{i}^{r}+I\right) \otimes(1+V)-(1+I) \otimes\left(y_{i}+V\right)\right)\left(\left(t_{j}^{r}+I\right) \otimes(1+V)\right. \\
& \left.-(1+I) \otimes\left(y_{j}+V\right)\right)-\left(\left(t_{j}^{r}+I\right) \otimes(1+V)-(1+I) \otimes\left(y_{j}+V\right)\right) \\
& \times\left(\left(t_{i}^{r}+I\right) \otimes(1+V)-(1+I) \otimes\left(y_{i}+V\right)\right)
\end{aligned}
$$




$$
\begin{aligned}
= & \left(t_{i}^{r} t_{j}^{r}+I\right) \otimes(1+V)+(1+I) \otimes\left(y_{i} y_{j}+V\right)-\left(t_{j}^{r}+I\right) \otimes\left(y_{i}+V\right)- \\
& -\left(t_{i}^{r}+I\right) \otimes\left(y_{j}+V\right)-\left(t_{i}^{r} t_{j}^{r}+I\right) \otimes(1+V)-(1+I) \otimes\left(y_{j} y_{i}+V\right)+ \\
& +\left(t_{j}^{r}+I\right) \otimes\left(y_{i}+V\right)+\left(t_{i}^{r}+I\right) \otimes\left(y_{j}+V\right) \\
= & (1+I) \otimes\left(y_{i} y_{j}+V\right)-(1+I) \otimes\left(y_{j} y_{i}+V\right) \\
= & (1+I) \otimes\left(\left[y_{i}, y_{j}\right]+V\right) .
\end{aligned}
$$

Logo, tomando produtos dos elementos $x_{i}^{p}+U, x_{i}^{q}-x_{i}+U$ e $\left[x_{i}, x_{j}\right]+U$, vemos que $\Phi$ é sobrejetora.

Além disso, para $m<p$,

$$
\begin{aligned}
\Phi\left(x_{i}^{m}+U\right) & =\Phi\left(x_{i}+U\right)^{m} \\
& =\left(\left(t_{i}^{r}+I\right) \otimes(1+V)-(1+I) \otimes\left(y_{i}+V\right)\right)^{m} \\
& =\sum_{k=0}^{m} \alpha_{k}\left(t_{i}^{r(m-k)}+I\right) \otimes\left(y_{i}^{k}+V\right)
\end{aligned}
$$

onde $\alpha_{k}=\left(\begin{array}{c}m \\ k\end{array}\right) \in K$. Como $m<p$, temos que $\alpha_{k} \neq 0$. Assim, por exemplo, para $0<m^{\prime}<q$ e $0<m<p$

$$
\begin{aligned}
\Phi\left(x_{i^{\prime}}^{p m^{\prime}} x_{i}^{m}\left[x_{j_{1}}, x_{j_{2}}\right]+U\right)= & \left(\left(t_{i}^{m^{\prime}}+I\right) \otimes(1+V)\right)\left(\sum_{k=0}^{m} \alpha_{k}\left(t_{i}^{r(m-k)}+I\right) \otimes\left(y_{i}^{k}+V\right)\right) \\
& \times\left((1+I) \otimes\left(\left[y_{j_{1}}, y_{j_{2}}\right]+V\right)\right) \\
= & \sum_{k=0}^{m} \alpha_{k}\left(t_{i^{\prime}}^{m^{\prime}} t_{i}^{r(m-k)}+I\right) \otimes\left(y_{i}^{k}\left[y_{j_{1}}, y_{j_{2}}\right]+V\right) .
\end{aligned}
$$

Note que $r(m-k)<q$ e $k<p$, isto é, $t_{i^{\prime}}^{m^{\prime}} t_{i}^{r(m-k)}+I$ pertence a base de $A$ e $y_{i}^{k}\left[y_{j_{1}}, y_{j_{2}}\right]+V$ pertence a base de $B$.

De um modo geral,

$$
\begin{gathered}
\Phi\left(x_{i_{1}^{\prime}}^{p m_{1}^{\prime}} \cdots x_{i_{k^{\prime}}^{\prime}}^{p m_{k^{\prime}}^{\prime}} x_{i_{1}}^{m_{1}} \cdots x_{i_{k}}^{m_{k}}\left[x_{j_{1}}, x_{j_{2}}\right] \cdots\left[x_{j_{2 l-1}}, x_{j_{2 l}}\right]+U\right)= \\
\sum_{\xi_{i_{1}}=0}^{m_{1}} \cdots \sum_{\xi_{i_{k}}=0}^{m_{k}}\left(\alpha t_{i_{1}^{\prime}}^{m_{1}^{\prime}} \cdots t_{i_{k^{\prime}}^{\prime}}^{m_{k^{\prime}}^{\prime}} t_{i_{1}}^{r\left(m_{1}-\xi_{i_{1}}\right)} \cdots t_{i_{k}}^{r\left(m_{k}-\xi_{i_{k}}\right)}+I \otimes y_{i_{1}}^{\xi_{i_{1}}} \cdots y_{i_{k}}^{\xi_{i_{k}}}\left[y_{j_{1}}, y_{j_{2}}\right] \cdots\left[y_{j_{2 l-1}}, y_{j_{2 l}}\right]+V\right) \\
\text { onde } 0 \neq \alpha \in K .
\end{gathered}
$$


Portanto, vemos que cada parcela do somatório no segundo membro da equação é unicamente determinada pelos monômios em $\mathcal{B}^{\prime}(\bmod U)$. Logo, as imagens dos monômios em $\mathcal{B}^{\prime}(\bmod U)$ pela $\Phi$ são linearmente independentes. Assim, $\Phi$ é injetora.

O seguinte lema pode ser encontrado em [28].

Lema 2. $x_{1}^{q p}-x_{1}^{p} \in T(E)$.

Pelo lema anterior, temos que $U \subseteq T(E)$. Com isso temos

Corolário 5.3. (Ver [3]) $T(E)$ é gerado como $T$-ideal por $\left[x_{1}, x_{2}, x_{3}\right]$ e $x_{1}^{q p}-x_{1}^{p}$, ou seja, $T(E)=U$.

Demonstração. Pelo que foi dito acima, resta mostra a inclusão $T(E) \subseteq U$. Seja $A^{(n)}$ a subálgebra de $A$ gerada por $t_{1}+I, \ldots, t_{n}+I$. Então, $A^{(n)} \subseteq \prod K$, onde $\prod K$ é um produto direto finito. De fato, sejam $T_{n}=\left\{t_{1}, \ldots, t_{n}\right\}$ e $K\left\langle T_{n}\right\rangle$ a álgebra livre de posto $n$. Então o conjunto de todos os homomorfismos de $K\left\langle T_{n}\right\rangle$ em $K$ é finito. Então seja,

$$
\begin{aligned}
& \Phi: K\left\langle T_{n}\right\rangle \longrightarrow \prod_{j=1}^{s} K \\
& \Phi(f)=\left(\phi_{1}(f), \ldots, \phi_{s}(f)\right)
\end{aligned}
$$

onde $s$ é o número de homomorfismos de $K\left\langle T_{n}\right\rangle$ em $K$. Logo, $f \in K \operatorname{Ker}(\Phi)$ se, e somente se, $f \in \operatorname{Ker}\left(\phi_{j}\right), j=1, \ldots, s$. Portanto, $\operatorname{Ker}(\Phi)=I$. Assim $A^{(n)}=K\left\langle T_{n}\right\rangle / I \simeq \operatorname{Im}(\Phi) \subseteq$ $\prod K \mathrm{e}$

$$
A^{(n)} \otimes B \subseteq\left(\prod K\right) \otimes B \simeq \prod(K \otimes B) \simeq \prod B
$$

Como $B$ satisfaz polinômios em $T(E)$, temos que $A^{(n)} \otimes B$ também satisfaz polinômios em $T(E)$. Mas como $n$ é arbitrário, $A \otimes B$ pertence a variedade determinada por $T(E)$, isto é, $T(E) \subseteq U$. 


\section{Referências Bibliográficas}

[1] Bekh-Ochir, C., Rankin, S.A.: Examples of associative algebras for which the T-space of central polynomials is not finitely based, Israel Journal of Mathematics 186 (2011), 333-347.

[2] Bekh-Ochir, C., Rankin, S.A.: The central polynomials of the infinite-dimensional unitary and nonunitary Grassmann algebras, Journal of Algebra and Its Aplications $9(5)$ (2010), 687-704.

[3] Bekh-Ochir, C., Rankin, S.A.: The identities and the central polynomials of the infinite dimensional unitary Grassmann algebra over a finite field, Communications in Algebra 39 (2011), 819-829.

[4] Belov, A.Ya.: Counterexamples to the Specht problem, Sbornik Mathematics 191 (2000), 329-340.

[5] Belov, A.Ya.: On non-Specht varieties, Fundamental'naya i Prikladnaya Matematika 5 (1999), 45-66 (in Russian).

[6] Brandão Jr., A., Koshlukov, P., Krasilnikov, A. Silva, E.A.: The central polynomials for the Grassmann algebra, Israel Journal of Mathematics 179 (2010), 127-144 .

[7] Centrone, L.: $\mathbb{Z}_{2}$-graded identities of the Grassmann algebra in positive characteristic, Linear Algebra and Applications 435 (2011), 3297-3313.

[8] Chiripov, P.Z., Siderov, P.N.: On bases for identities of some varieties of associative algebras (Russian), PLISKA Studia Mathematica Bulgarica 2 (1981), 103-115.

[9] Deryabina, G., Krasilnikov, A.: The subalgebra of graded central polynomials of an associative algebra, Journal of Algebra 425 (2015), 313-323.

[10] Drensky,V.: Free algebras and PI-algebras, Graduate Course in Algebra, Springer, Singapore, 1999. 
[11] Drensky,V., Formanek, E.: Polynomial identity rings, Advanced Courses in Mathematics, CMR Barcelona, Birkhäuser Verlag, Basel, 2004.

[12] Giambruno, A., Koshlukov, P.: On the identities of the Grassmann algebra in characteristic $p>0$, Israel Journal of Mathematics 122 (2001), 305-316.

[13] Giambruno, A., Zaicev, M.: Polynomial identities and asymptotic methods, Mathematical Surveys and Monographs, 122, American Mathematical Society, Providence, RI, 2005.

[14] Gonçalves, D.J., Krasilnikov, A., Sviridova, I.: Limit T-subspaces and the central polynomials in $n$ variables of the Grassmann algebra, Journal of Algebra, 371 (2012), 156-174.

[15] Gonçalves, D.J., Krasilnikov, A., Sviridova, I.: Limit T-subalgebras in free associative algebras, Journal of Algebra, 412 (2014), 264-280.

[16] Grishin, A. V.: Examples of T-spaces and T-ideals of characteristic 2 without the finite basis property, Fundamental'naya i Prikladnaya Matematika 5 (1999), 101-118 (in Russian).

[17] Grishin, A. V.: On the structure of the centre of a relatively free Grassmann algebra, Russian Mathematical Surveys 65 (2010) 781-782.

[18] Grishin, A. V.: On non-Spechtianness of the variety of associative rings that satisfy the identity $x^{32}=0$, Electronic Research Announcements of the American Mathematical Society 6 (2000), 50-51.

[19] Grishin, A. V., Tsybulya, L.M.: On the multiplicative and T-space structure of the relatively free Grassmann algebra, Sbornik Mathematics 200 (2009), 1299-1338.

[20] Kanel-Belov, A., Karasik, Y, Rowen, L.H.: Computational aspects of polynomial identities, Monographs and Research Notes in Mathematics, 1, Taylor and Francis, New York-London, 2016.

[21] Kemer, A.R.: Finite basability of identities of associative algebras, Algebra and Logic 26(5) (1987), 362-397.

[22] Kemer, A.R.: Ideals of identities of the associative algebras, Translations of Mathematical Monographs, vol 87, American Mathematical Society, Providence, RI, 1991. 
[23] Kemer, A.R.: Varieties and $\mathbb{Z}_{2}$-graded algebras, Mathematics of the USSR-Izvestiya 25 (1985), 359-374.

[24] Kireeva, E.A., Krasilnikov, A.N.: On some extremal varieties of associative algebras, Mathematical Notes, 78 (2005), 503-517.

[25] Krakowski, D., Regev, A.: The polynomial identities of the Grassmann algebra, Transactions of the American Mathematical Society, 181 (1973), 429-438.

[26] Latyshev, V.N., On the choice of basis in a T-ideal, Sibirskii Matematicheskii Zhurnal 4(5) (1963), 1122-1126 (in Russian).

[27] Razmyslov, Yu. P. : Identities of algebras and their representations, Translations of Mathematical Monographs, 138, American Mathematical Society, Providence, RI, 1994.

[28] Regev, A.: Grassmann algebras over finite fields, Communications in Algebra, 19 (1991), 1829-1849.

[29] Rowen, L.W.: Polynomial identities in ring theory, Pure and Applied Mathematics, 84, Academic Press, New York-London, 1980.

[30] Shchigolev, V. V.: Examples of T-spaces with an infinite basis, Sbornik Mathematics 191 (2000), 459-476.

[31] Shchigolev, V. V.: Examples of infinitely based T-ideals, Fundamental'naya i Prikladnaya Matematika 5 (1999), 307-312 (in Russian).

[32] Shchigolev, V. V.: Construction of non-finitely based T-ideals, Communications in Algebra 29 (2001), 3935-3941.

[33] Specht, W.: Gezetze in Rigen, Mathematische Zeitschrift 52 (1950), 557-589.

[34] Stojanova-Venkova, A.H.: Bases of identities of Grassmann algebras, Serdica 6, 1 (1980), 63-72 (in Russian).

[35] Silva, E.A.: Polinômios centrais em algumas álgebras associativas e representações de grupos. Tese de Doutorado, UnB, 2008.

[36] da Silva, V.R.T., Di Vincenzo, O.M.: On $\mathbb{Z}_{2}$-graded polynomial identities of the Grassmann algebra, Linear Algebra and Applications 431 (2009), 56-72.

[37] Tsybulya, L. M.: Theorems on equalization and monomiality in a relatively free Grassmann algebra, Journal of Mathematical Sciences, 163(6) (2009), 759-773. 